\title{
Representing equilibrium aggregates in aggregate games with applications to common agency
}

\author{
David Martimort ${ }^{\mathrm{a}}$, Lars Stole ${ }^{\mathrm{b}, *}$ \\ a Paris School of Economics-EHESS, France \\ ${ }^{\mathrm{b}}$ University of Chicago, Booth School of Business, United States
}

\section{A R T I C L E I N F O}

\section{Article history:}

Received 5 May 2010

Available online 30 August 2012

\section{JEL classification:}

D82

\section{Keywords:}

Aggregate games

Common agency games

Supply-function games

\begin{abstract}
A B S T R A C T
An aggregate game is a normal-form game with the property that each player's payoff is a function of only his own strategy and an aggregate of the strategy profile of all players. Such games possess properties that can often yield simple characterizations of equilibrium aggregates without requiring that one solves for the equilibrium strategy profile. When payoffs have a quasi-linear structure and a degree of symmetry, we construct a selfgenerating maximization program over the space of aggregates with the property that the solution set corresponds to the set of equilibrium aggregates of the original $n$-player game. We illustrate the value of this approach in common-agency games where the players' strategy space is an infinite-dimensional space of nonlinear contracts. We derive equilibrium existence and characterization theorems for both the adverse selection and moral hazard versions of these games.
\end{abstract}

(c) 2012 Elsevier Inc. All rights reserved.

\section{Introduction}

An aggregate game is a normal-form game with the property that each player's payoff is a function of only his own strategy and an aggregate function of the strategy profile of all players. The Cournot quantity game is probably the best known example of such a setting (the aggregate is the industry supply) and was the motivation behind Selten's (1970) initial treatment of aggregate games. More generally, this class of games includes a variety of strategic settings such as public goods games, common resource games, rent-seeking contests, cost-sharing games, team games, persuasion games and patent races. ${ }^{1}$ Results on the existence of equilibria and comparative statics properties are available in many of these games if one makes additional assumptions on the structure of the strategy spaces and on the relationship between the aggregate and each player's strategy in the payoff functions. ${ }^{2}$ That said, to obtain strong results, previous papers on aggregate games have

\footnotetext{
t An older version of this paper was circulated under the title of "A note on aggregate-strategy games", 2005. The authors thank John Birge, Matt Jackson, Emir Kamenica, David Myatt, Roger Myerson, David Rahman, Phil Reny, Michael Schwarz and participants at the "Workshop on Games with an Aggregative Structure" held at University of Strathclyde, Glasgow for helpful conversations. The comments by an Associate Editor, Matt Jackson and two additional referees were instrumental in helping us reshaping our ideas on this paper. All errors are ours.

* Corresponding author.

E-mail addresses: david.martimort@parisschoolofeconomics.eu (D. Martimort), lars.stole@chicagobooth.edu (L. Stole).

1 See Bergstrom et al. (1986), Okuguchi (1993), Corchon (1994), Cornes and Hartley (2000, 2005), Acemoglu and Jensen (2009) and Jensen (2010), and the many references cited therein for examples of these games. See Gentzkow and Kamenica (2011) for a study of persuasion games.

2 See in particular, Corchon (1994), Dubey et al. (2006), and Acemoglu and Jensen (2009) for comparative statics results; existence results can be found in Szidarovszky and Yakowitz (1977), Novshek (1984, 1985), Kukushkin (1994, 2004), Dubey et al. (2006), and Jensen (2010).
} 
largely restricted attention to games with finite-dimensional strategy spaces and, more often than not, a single-dimension aggregate space. ${ }^{3}$

In the games that are of direct interest to the present authors (e.g., competitive agency games with nonlinear transfer functions), strategies are most naturally modelled as infinite-dimensional objects. Nonetheless, this larger class of aggregate games shares a set of algebraic properties with the simpler games that can often provide immediate characterizations of equilibrium aggregates without requiring that one solves for the equilibrium strategy profile. We demonstrate that the defining nature of payoffs in an aggregate game allows one to embed the strategic analysis into the aggregate-strategy space and, if payoffs also satisfy a form of quasi-linearity, that such an embedding converts an $n$-player game to a simpler object - a single-person, self-generating maximization program. The proofs of these results are straightforward, following directly from the algebraic properties of the players' payoffs. Nevertheless, this simple projection of an aggregate game into the space of aggregate strategies may yield a characterization of the set of equilibrium aggregates without the need for an explicit construction of equilibrium strategies. Moreover, equilibrium existence may be derived directly from these aggregate representations.

In addition to proving that the set of equilibrium aggregates is non-empty, for a large class of aggregate games (aggregate games with quasi-linear payoffs and a bijective aggregate function), we are able to infer properties of the equilibrium aggregate set by studying only the aggregate maximization program without calculating an equilibrium strategy profile. This is our main result in Section 2 for the class of quasi-linear aggregate games. Representing the equilibrium aggregates with a maximization program also provides a neutrality result as a corollary: The set of aggregate equilibrium outcomes is invariant with respect to variations in individual payoff functions, provided that the sum of the players' payoffs remain fixed.

Our approach which characterizes equilibrium aggregates as the solution set to a particular kind of self-generating maximization program is particularly useful for proving equilibrium existence in contracting games in which competing principals use incentive contracts to influence an intrinsic ${ }^{4}$ common agent. In the second part of the paper (Section 3 ), we use the general result in Section 2 to provide a detailed characterization of the equilibrium aggregates in such common agency games - both in settings with adverse selection and with moral hazard.

When the agent has private information at the time of contracting (adverse selection), an aggregate representation provides a simple proof of equilibrium existence. This proof is accomplished by constructing an aggregate maximization program whose solution set corresponds to the set of equilibrium aggregates and then applying standard arguments for the existence of optimizers. The ability to prove existence through mathematical programming arguments (rather than fixed point arguments) provides a significant improvement over the existing literature. Indeed, the existence of equilibria under intrinsic common agency has so far received only scarce attention. Taking existence as given, a first branch of that literature was mostly directed towards unveiling general properties of equilibria, with a particular focus on the validity and scope of the Revelation and Delegation Principles (Peters, 2001; Martimort and Stole, 2002; Page and Monteiro, 2003). Other papers derived existence in specific settings by explicitly computing or characterizing equilibria. Along those lines, Stole (1991) and Martimort (1992) characterized equilibria for games of private agency (each principal contracts on a specific activity of their common agent) whereas Martimort and Stole (2010) focused on public agency (all principals contract on the same variables). The existence of equilibrium in more general menu games, or more precisely for their mixedstrategy extensions, was investigated by Page and Monteiro (2003), Monteiro and Page (2005), Page (2008) and Carmona and Fajardo (2009). While the first contributions take as fixed the strategy by the common agent, Carmona and Fajardo (2009) determined this strategy endogenously as part of the solution concept. ${ }^{5}$ Our approach provides existence of a purestrategy equilibrium in a public agency game and allows us to reconstruct from the aggregate the individual strategies for each principal. Furthermore, the general neutrality result presented in Section 2 can be applied to common agency games with adverse selection, yielding the remarkable conclusion that only the aggregate of the principals' payoff functions matters for determining the aggregate equilibrium behavior of the principals; the specific composition of the principals' payoffs in forming the aggregate is immaterial.

When the contractual imperfection is moral hazard rather than adverse selection, we show the existence of an equilibrium in a game in which a risk neutral agent is protected by limited liability while contracting with several principals. This environment complements earlier works on the topic of moral hazard and common agency which have obtained existence under rather stringent conditions involving linear contracts and CARA-Gaussian models as in Holmström and Milgrom (1988) and Dixit (1996) or discrete distributions with risk aversion but a finite number of effort levels as in Bernheim and Whinston (1986a) and Fraysse (1993). As with the adverse selection contracting game, an interesting neutrality result is immediate from the aggregate representation.

In Section 2 we provide general definition for the class of aggregate games. Placing additional quasi-linear structure on payoffs, we derive a sharp necessary and sufficient condition for equilibrium aggregates which can be interpreted as the solution set to a self-generating maximization program. Illustrating the power and simplicity of the aggregate game

\footnotetext{
${ }^{3}$ Jensen (2010) and Acemoglu and Jensen (2009) briefly consider games with multi-dimensional aggregates, but the comparative statics and existence results are compromised by the requirement of supermodularity and decreasing differences that are less likely to be satisfied in many applications.

4 Following Bernheim and Whinston (1986a), common agency is intrinsic if the agent must either contract with every principal in common or remain inactive in the game.

5 This requires an argument on endogenous sharing rule along the lines of Simon and Zame (1990).
} 
techniques, we apply the results for this class of aggregate games to common-agency contracting games both under adverse selection or moral hazard and limited liability in Section 3.

\section{Aggregate games}

\subsection{General definitions}

An aggregate game is a normal-form game with the additional requirement that each player $i$ 's payoff depends only upon his own strategy, $x_{i} \in X$, and a linear aggregate of the full strategy profile, $y=\sum_{j \in N} x_{j}$, where $\mathbf{x}=\left(x_{1}, \ldots, x_{n}\right) \in(X)^{n}=\mathbf{X}$. We assume that the strategy space $X$ is closed under addition, from which it follows that $y \in X$ and the domain of each player's payoff function is $X \times X .^{6}$ Formally,

Definition 1. An aggregate game, denoted $G=\left\{X,\left(u_{i}\right)_{i \in N}\right\}$, is a normal-form game of $n$ players, indexed by $i \in N \equiv\{1, \ldots, n\}$, in which each player $i$ chooses a strategy $x_{i} \in X \subseteq \mathcal{V}$ where $(\mathcal{V},+, \cdot)$ is a real linear space, and each player $i$ has preferences over strategy profiles representable by a payoff function, $u_{i}: X \times X \rightarrow \mathbb{R}$, which maps player $i$ 's strategy, $x_{i}$, and the aggregate, $y=\sum_{j \in N} x_{j}$, to the payoff $u_{i}\left(x_{i}, y\right)$.

An equilibrium of $G$ is a profile of strategies, $\overline{\mathbf{x}} \in \mathbf{X}$, such that for each $i \in N$,

$$
u_{i}\left(\bar{x}_{i}, \bar{x}_{i}+\sum_{j \neq i} \bar{x}_{j}\right) \geqslant u_{i}\left(x_{i}, x_{i}+\sum_{j \neq i} \bar{x}_{j}\right), \quad \forall x_{i} \in X,
$$

and the associated equilibrium aggregate is denoted $\bar{y}=\sum_{j \in N} \bar{x}_{j} \in X$. The (possibly empty) equilibrium set for game $G$ is denoted $\mathcal{E}(G) \subseteq \mathbf{X}$ and the corresponding equilibrium aggregate set is denoted $\mathcal{E}_{Y}(G) \subseteq X$.

To illustrate the definitions, we provide simple, concrete examples of aggregate games. We begin with the simplest example in the literature - the Cournot output game with one-dimensional strategy spaces.

Example 1 (Cournot games). Each of the $n$ firms simultaneously produces an output, $q_{i} \geqslant 0$, at a cost of $C_{i}\left(q_{i}\right)=c_{i} q+F_{i}$ if $q_{i}>0$ and 0 otherwise. The common strategy space is $X=\mathbb{R}_{+}$. The market price is set such that total demand, $D(p)$ (with inverse demand $P(Q)$ ), is equal to the aggregate market supply $\sum_{i \in N} q_{i}=Q$. Preferences for player $i$ are given by:

$$
u_{i}\left(q_{i}, Q\right)= \begin{cases}q_{i}\left(P(Q)-c_{i}\right)-F_{i} & \text { if } q_{i}>0 \\ 0 & \text { if } q_{i}=0\end{cases}
$$

Example 1 allows us to showcase an important property that plays a role in the sequel: although $X=\mathbb{R}_{+}$is closed under addition, it is not closed under subtraction: i.e., the difference of two positive outputs is not necessarily positive itself. In this case, $X=\mathbb{R}_{+}$is not a linear subspace of $\mathcal{V}=\mathbb{R}$. If, however, $X$ is closed under both addition and subtraction, then any player $i$ facing a profile of strategies $\mathbf{x}_{-i}$ can implement any aggregate $y \in X$ by choosing $x_{i}=y-\sum_{j \neq i} x_{j}$. In such a case, every player has the freedom to manipulate the aggregate. When this property holds in an aggregate game, we say that the aggregate is bijective. ${ }^{7}$ Such bijectivity plays an important role in the sequel.

Our second example of supply-function games differs from the simple Cournot setting in two respects: the aggregate in such games is bijective and the strategy spaces are infinite-dimensional.

Example 2 (Supply-function games). Consider the supply-function game studied in Klemperer and Meyer (1989). Suppose that each of $n$ firms chooses a supply function as its strategy, $S_{i}: \mathbb{R}_{+} \mapsto \mathbb{R}$, where $S_{i}$ is a bounded function on the domain of nonnegative prices mapping to the number of units firm $i$ commits to supply at the price $p$. We allow that supply commitments may be negative, which has the interpretation of a net demand by the firm. Each firm chooses its supply function, $S_{i}$, prior to knowing the state of demand, $\theta$, which is distributed over a set $\Theta$ according to the distribution $F$. We denote demand in state $\theta$ as $D(p, \theta)$. After the supply functions are chosen and $\theta$ is realized, a market maker chooses the price $p(\theta, S)$ to equate supply to demand: $\sum_{i \in N} S_{i}(p(\theta, S))=D(p(\theta, S), \theta) .{ }^{8}$ The relevant aggregate in this game is the

\footnotetext{
6 In an earlier version of this work, we considered a more general setting in which $x_{i} \in X_{i}$ for $i=1, \ldots, n$, and the aggregate is $y=\phi(\mathbf{x})$ where $\phi$ is a proper (i.e., not one-to-one) aggregate function, $\phi: \mathbf{X} \rightarrow Y$, where $Y=\phi(\mathbf{X})$.

7 In a previous version of this paper we allowed for more general aggregate functions of the form $y=\phi(\mathbf{x})$, where $\phi: X_{1} \times \cdot \times X_{n} \rightarrow Y$. In this case, the appropriate generalization of bijectivity is that for every player $i$ and every profile $\mathbf{x}_{-i} \in \mathbf{X}_{-i}$, the mapping $x_{i} \mapsto \phi\left(x_{i}, \mathbf{x}_{-i}\right)$ is bijective between $X_{i}$ and $Y$, holding $\mathbf{x}_{-i}$ fixed. In the present case, $\phi(\mathbf{x})=\sum_{i} x_{i}$ and $X_{1}=\cdots=X_{n}=Y=X$, so this requirement reduces to the requirement that $X$ is closed under addition and subtraction.

8 To simplify the analysis, we assume that such solution is unique.
} 
market supply function, $S(p)=\sum_{i \in N} S_{i}(p)$. Assuming that all firms have the same constant marginal cost $c$, preferences for player $i$ are represented by the payoff function

$$
u_{i}\left(S_{i}, S\right)=\int_{\Theta} S_{i}(p(\theta, S))(p(\theta, S)-c) d F(\theta) .
$$

Multi-principal games with contract competition for a common agent also offer examples of aggregate games with infinite-dimensional strategy spaces. These examples are of prime importance in view of the existence and invariance results we provide using aggregate techniques in Section 3 below.

Example 3 (Intrinsic common-agency games with adverse selection). Each of $n$ principals offers a nonlinear contract, $T_{i}, i=$ $1, \ldots, n$, which is a commitment by principal $i$ to pay a common agent $T_{i}(q)$ conditional on the agent's choice of $q \in \mathcal{Q}$, from a compact set. The agent's preferences, however, are private information at the time of contracting, which we parameterize by a privately-known preference parameter, $\theta$, which is distributed over a set $\Theta$ according to the distribution $F$. Given the presence of private information, each principal will typically gain from distorting the choice of $q$ in order to capture some of the agent's information rent. In this paper, we assume that common agency is intrinsic in the game: the agent must either accept all contract offers, accepting the aggregate transfer $T(q)=\sum_{i \in N} T_{i}(q)$, or reject all contracts and obtaining a reservation payoff normalized to zero. An agent of type $\theta$ has a bounded, continuous benefit function, $u(\cdot, \theta): \mathcal{Q} \rightarrow \mathbb{R}$, and a net payoff

$$
u(q, \theta)+\sum_{i \in N} T_{i}(q)
$$

upon accepting all of the contracts and choosing $q \in \mathcal{Q}$. The agent's optimal choice of $q$ will be a function of $\theta$ and the aggregate transfer function, $T=\sum_{i} T_{i}$. Indeed, for any upper-hemicontinuous aggregate contract offer, $T=\sum_{i \in N} T_{i}$, the agent's best-response correspondence is non-empty, compact-valued and upper-hemicontinuous. Denote the correspondence by

$$
Q_{0}(\theta, T) \equiv \underset{q \in Q}{\arg \max } u(q, \theta)+T(q),
$$

and choose $q(\theta, T) \in Q_{0}(\theta, T)$ to be some measurable selection. Denoting principal $i$ 's gross surplus by $v_{i}(q)$, his expected payoff is

$$
u_{i}\left(T_{i}, T\right)=\int_{\Theta}\left(v_{i}(q(\theta, T))-T_{i}(q(\theta, T))\right) d F(\theta) .
$$

Note that principals are allowed to demand payments from the agent (i.e., transfers may be negative). This is an important ingredient to ensure that the aggregate in the game is bijective. We examine this game further in Section 3.1. ${ }^{9}$

Example 4 (Intrinsic common-agency games with moral hazard). Similar to the adverse selection setting, each of $n$ principals non-cooperatively offers a nonlinear contract, $T_{i}, i=1, \ldots, n$, which commits principal $i$ to pay the common agent $T_{i}(q)$ for any realization of output $q \in \mathcal{Q}=\left[q_{0}, q_{1}\right]$. The cumulative distribution of output is given by $F(q \mid e)$, with positive density $f(q \mid e)$ on $\mathcal{Q}$, where $e \in[0, \bar{e}]$ is the level of the agent's privately-observed, costly effort. We assume that the agent is risk neutral and his preferences over transfers and effort can be represented as

$$
\int_{\mathcal{Q}} T(q) d F(q \mid e)-\psi(e)
$$

where $\psi$ is the agent's increasing, convex disutility of effort satisfying $\psi(0)=\psi^{\prime}(0)=0$. In the intrinsic common-agency version of this game, the agent either accepts the aggregate contract $T=\sum_{i} T_{i}$ or refuses all offers and chooses $e=0$. Thus, the agent's optimal effort $e$ will be a function of the aggregate transfer function, $T$. Denote $e(T)$ as the equilibrium choice of the agent - a selection from the agent's optimal effort correspondence:

\footnotetext{
9 When the agent can choose a proper subset of principals with which to contract, we say that the choice of common agency is no longer intrinsic (to the game) but delegated (to the agent). Formally, it is without loss of generality to model a game of delegated agency as an intrinsic common agency game, except with the additional requirement that each principal $i \in N$ offers a non-negative payment schedule, $T_{i} \geqslant 0$. Such games share with the Cournot example the fact that the aggregate mappings are not bijective - the difference between two non-negative payment schedules may not be non-negative. The analysis of such delegated common agency games requires, in addition to the aggregate tools of the present paper, a set of new techniques using non-smooth analysis. This analysis is developed in Martimort and Stole (2011).
} 


$$
e(T) \in \underset{e \in[0, \bar{e}]}{\arg \max } \int_{\mathcal{Q}} T(q) d F(q \mid e)-\psi(e) .
$$

If the agent is protected by limited liability (normalized to zero), the equilibrium aggregate offer will be non-negative. Even so, because principals are allowed to demand payments from the agent, individual transfers may be negative. Denoting again principal $i$ 's gross surplus by $v_{i}(q)$, his preferences are given by:

$$
u_{i}\left(T_{i}, T\right)=\int_{\Theta}\left(v_{i}(q)-T_{i}(q)\right) d F(q \mid e(T)) .
$$

We examine this game further in Section 3.2.

\subsection{Quasi-linear aggregate games}

Examples 2, 3 and 4 exhibit a key feature beyond their aggregate structure which is an important ingredient in using an aggregate maximization program to characterize the equilibrium set: Payoffs are quasi-linear in $x_{i}$ (i.e., they are linear in $x_{i}$ holding the aggregate $y$ fixed). In addition, a degree of payoff symmetry between players is present. In the case of supplyfunction games, each firm's profit as a function of $p$ is given by $(p-c) S_{i}(p)$, and thus each firm has the same coefficient on its individual strategy, $S_{i}$. In the case of common agency, the coefficient on each principal's transfer, $T_{i}$, is identical and equal to one (although the individual returns to $q$ through $v_{i}(q)$ may be quite different across principals). Our definition of quasi-linear aggregate games will incorporate a weak notion of symmetric preferences which is sufficient for our main characterization result. ${ }^{10}$

We proceed by first defining a large subclass of aggregate games with a quasi-linear payoff structure that satisfies a requisite degree of symmetry. In view of applying our general results to games whose strategy spaces are infinite-dimensional, our definition includes the case in which payoffs are defined over infinite-dimensional vector spaces. Together with the requirement of quasi-linearity, this is accomplished by representing payoffs by means of bilinear forms.

A bilinear form is a real function which combines elements from two linear spaces, which we denote as $\langle\cdot, \cdot\rangle: \mathcal{V} \times \mathcal{W}$ $\rightarrow \mathbb{R}$, with the defining property that for every $f \in \mathcal{V},\langle f, \cdot\rangle$ is a linear functional on $\mathcal{W}$ and similarly $\langle\cdot, g\rangle$ is a linear functional on $\mathcal{V} .{ }^{11}$ As an example, for common agency games we will take $\mathcal{V}$ to be the space of bounded Borel-measurable functions, $\mathcal{W}$ to be the space of distributions, and the bilinear form to be the Lebesgue-Stieltjes integral of a function, $f \in \mathcal{V}$, with respect to the measure derived from the distribution $g \in \mathcal{W}$; i.e., $\langle f, g\rangle \equiv \int f d g$. Presently, however, we use the bilinear form more abstractly to define payoffs for the class of quasi-linear aggregate games.

Definition 2. A game $G$ is a quasi-linear aggregate game if it is an aggregate game in which the preferences for each player $i \in N$ are representable by $u_{i}: X \times X \rightarrow \mathbb{R}$,

$$
u_{i}\left(x_{i}, y\right)=\alpha(y)+\left\langle x_{i}+\gamma_{i}, \beta(y)\right\rangle
$$

where $\alpha: X \rightarrow \mathbb{R}, \beta: X \rightarrow \mathcal{W}$ and $\gamma_{i} \in \mathcal{V}$.

Thus defined, the class of quasi-linear aggregate games requires a particular form of payoff symmetry: for a fixed aggregate, $y$, the difference in payoffs to player $i$ between any two strategies, $x$ and $x^{\prime}$, is the same as the difference in payoff to player $j$ for the same two strategy choices:

$$
u_{i}(x, y)-u_{i}\left(x^{\prime}, y\right)=u_{j}(x, y)-u_{j}\left(x^{\prime}, y\right)=\left\langle x-x^{\prime}, \beta(y)\right\rangle .
$$

Note that this requirement does not require that each player $i$ has identical preference orderings over $X \times Y$ as the components $\gamma_{i} \in \mathcal{V}$ may vary across players introducing preferences asymmetries over the impact of $\beta(y)$. Moreover, the quasi-linear payoff restriction also allows for nonlinearities in $y$ (and thus indirectly in $x_{i}$ ). Finally, it is worth noting that we do not require that quasi-linear aggregate games have bijective aggregates, although we will use these assumptions below to establish the sufficiency portion of our main characterization result.

Example 1 (Cournot games, continued). The class of quasi-linear aggregate games contains many interesting applications, including the Cournot game with no fixed costs

$$
u_{i}(q, Q)=q_{i}(P(Q)-c)
$$

\footnotetext{
10 In an early version of this paper we emphasized that the necessary conditions for equilibrium aggregates can be proven for a weaker notion of payoff symmetry than what is used below. In the present paper, we use a stronger notion throughout to simplify the presentation and proofs.

11 An inner product is an example of a bilinear form that is also symmetric $(\mathcal{V}=\mathcal{W},\langle f, g\rangle=\langle g, f\rangle)$. In the simplest such case, $\mathcal{V}=\mathcal{W}=\mathbb{R}$ and the bilinear form reduces to scalar multiplication. A bilinear form, however, is more general than the inner product, allowing asymmetries and semi-definiteness. E.g., $\langle f, g\rangle=\left\langle f^{\prime}, g\right\rangle$ for $g \neq \mathbf{0}_{W}$ need not imply that $f=f^{\prime}$.
} 
Cournot games with non-constant marginal costs would yield payoff functions that are not affine in each player's own strategy (holding the aggregate output constant); Cournot games with positive fixed-costs are not quasi-linear because of the discontinuities at zero. Of course, the strategy space in a Cournot game is not bijective.

Examples 2, 3, 4 (Distributional aggregate games). The common-agency contracting games which motivated this paper are examples of quasi-linear aggregate games with infinite-dimensional strategy spaces in which the aggregate enters payoff functions via a probability distribution. Since this class also contains other interesting aggregate games such as supplyfunction games, we define the class more inclusively and refer to such strategic settings as distributional aggregate games for short. In distributional aggregate games, players choose strategies from subset of real bounded, Borel-measurable functions, $X \subseteq \mathcal{B}_{b}(\Omega)$, defined on some $\Omega$, and for which the aggregate function, $y=\sum_{i} x_{i}$, determines a probability distribution on $\Omega$ given by $H(\cdot \mid y): \Omega \rightarrow[0,1]$. Choosing $\langle f, g\rangle=\int f d g$ as the bilinear form, the requirement of quasi-linearity implies that preferences are representable by

$$
u_{i}\left(x_{i}, y\right)=\alpha(y)+\int_{\Omega}\left(x_{i}(\omega)+\gamma_{i}(\omega)\right) d H(\omega \mid y),
$$

where $\alpha: X \rightarrow \mathbb{R}, \gamma_{i} \in \mathcal{B}_{b}(\Omega)$, and the integral is computed with respect to the Lebesgue-Stieltjes measure $H .^{12}$ Take for instance the case of supply-function games. For any aggregate-supply function, $S$, the distribution of demand states induces a well-defined probability distribution over prices, $p \in \mathcal{P}$ :

$$
H(p \mid S)=\operatorname{Prob}(\theta \in \Theta \mid p(\theta, S) \leqslant p) .
$$

Firm $i$ 's expected profit function takes the form in (3):

$$
u_{i}\left(S_{i}, S\right)=\int_{\mathcal{P}} S_{i}(p)(p-c) d H(p \mid S)
$$

In the case of intrinsic common-agency games, the expressions of the principals' payoffs written in terms of distributions induced by an aggregate strategy have a similar structure and are presented in Section 3.

\subsection{Aggregate representation}

In this section we derive our main aggregate equilibrium characterization for the class of quasi-linear aggregate games.

\subsubsection{Necessary conditions for $\mathcal{E}_{Y}(G)$}

We begin by finding necessary conditions for equilibrium aggregates without assuming that the aggregate is bijective or that the game is quasi-linear. To this end, observe that a strategically irrelevant change of variables allows us to restate a player's optimal choice of strategy in terms of an optimal choice of the aggregate:

Lemma 1 (Aggregate concurrence). For any aggregate game G,

$$
\bar{x}_{i} \in \underset{x_{i} \in X}{\arg \max } u_{i}\left(x_{i}, x_{i}+\sum_{j \neq i} \bar{x}_{j}\right) \Longleftrightarrow \bar{y} \in \underset{y \in X+\sum_{j \neq i} \bar{x}_{j}}{\arg \max } u_{i}\left(y-\sum_{j \neq i} \bar{x}_{j}, y\right) .
$$

An immediate implication is that the equilibrium aggregate, $\bar{y}$, must lie in the aggregate best-response correspondence of every player (i.e., in the intersection of the players' aggregate best-response correspondences). All players must agree on the choice of the aggregate in equilibrium, to the extent that any individual player can affect it. This principle of aggregate concurrence, whose insight goes back at least to Selten (1970), provides a powerful perspective for analyzing equilibria in aggregate games. ${ }^{13}$

From the principle of aggregate concurrence, it follows that an equilibrium aggregate must maximize the "welfare" function obtained by summing the players' payoffs where each player $i$ 's strategy $x_{i}$ is replaced with $y-\sum_{j \neq i} \bar{x}_{j}$. In the case in which $G$ is a quasi-linear aggregate game, this collective surplus function is simply

\footnotetext{
12 Because $H$ is a probability distribution (and therefore of bounded variation and right-continuous), this integral is well defined over the set of measurable, bounded functions. See, for example, Aliprantis and Border (2006, Theorem 11.8). Note also that $H$ may be discontinuous; indeed such discontinuities may arise naturally in equilibrium. To anticipate results which follow, in the case of common agency under adverse selection, $H$ is discontinuous at any $q \in \mathcal{Q}$ which is chosen by a positive measure of agents (i.e., at all points of bunching).

13 While others have used this idea indirectly, to our knowledge Bernheim and Whinston (1986a) are the first to recognize explicitly the force of this principle in their study of moral hazard in a common agency game with finite-dimensional strategies: "We underscore the need to make the principals' objectives congruent in equilibrium: since all principals can effect the same changes in the aggregate incentive scheme, none must find any such change worthwhile". (Bernheim and Whinston, 1986a, p. 929.)
} 


$$
\frac{1}{n} \sum_{i \in N} u_{i}\left(y-\sum_{j \neq i} \bar{x}_{j}, y\right)=\Lambda(y, \bar{y})
$$

where

$$
\Lambda(y, \bar{y}) \equiv \alpha(y)+\left\langle y-\frac{n-1}{n} \bar{y}+\hat{\gamma}, \beta(y)\right\rangle
$$

and $\hat{\gamma} \equiv \frac{1}{n} \sum_{i} \gamma_{i}$

Importantly, the aggregate objective function $\Lambda(y, \bar{y})$ does not depend on the profile $\overline{\mathbf{x}}$ but only on their sum $\bar{y} .{ }^{14}$ This leads us to the following necessary condition which must be satisfied by an aggregate $\bar{y}$ in any equilibrium of a symmetric, quasi-linear aggregate game.

Lemma 2. For any quasi-linear aggregate game, $G$,

$$
\bar{y} \in \mathcal{E}_{Y}(G) \Longrightarrow \bar{y} \in \underset{y \in \bigcap_{i \in N} X+\left\{\bar{y}-\bar{x}_{i}\right\}}{\arg \max } \Lambda(y, \bar{y}) .
$$

Although the various instances of $\overline{\mathbf{x}}_{-i}$ in the individual players' objectives have been eliminated when moving to the aggregate objective function, $\Lambda$, they still have a potential role in defining the domain of optimization, $\bigcap_{i \in N} X+\left\{\bar{y}-\bar{x}_{i}\right\}$. If the aggregate is also bijective, however, then $X+\left\{\bar{y}-\bar{x}_{i}\right\}=X$ for all $i$ and the program in (6) simplifies accordingly. To summarize thus far, when $G$ is a quasi-linear aggregate game with a bijective aggregate, we conclude

$$
\bar{y} \in \mathcal{E}_{Y}(G) \Longrightarrow \bar{y} \in \underset{y \in X}{\arg \max } \Lambda(y, \bar{y}) .
$$

Notice that the maximization program on the right-hand side of (6) is relative to a fixed point. We refer to this type of problem as a self-generating maximization (SGM) program. When such an aggregate representation of the game is available it can be quite informative about $\mathcal{E}_{Y}(G)$ as we will illustrate in our common agency applications in Section 3. In particular, such program might allow useful conclusions about equilibria to be drawn directly from the maximization program without calculating equilibrium profiles, simply by computing aggregates that solve this problem. ${ }^{15}$

Characterizing the solution set to the SGM programs above is often more tractable than explicitly calculating the set of equilibria. Instead of looking for a fixed point over $n$-tuples of strategy profiles (i.e., $\overline{\mathbf{x}} \in X^{n}$ ), the aggregate analysis can be reduced to looking for a fixed-point in an optimization program over aggregates $(\bar{y} \in X){ }^{16}$

\subsubsection{Sufficient conditions for $\mathcal{E}_{Y}(G)$}

Condition (7) states that membership in the solution set of a particular SGM program is a necessary condition for any equilibrium aggregate. While useful, this does not tell us which solutions to the SGM program (if any) represent equilibrium aggregates. To complete the characterization, we now demonstrate by construction that any aggregate that is a solution to the SGM program is an aggregate in some equilibrium. To this end, we will rely on the hypotheses of quasi-linear payoffs and the bijectivity of the aggregate.

Suppose that $\bar{y}$ is a solution to the SGM program. Construct the strategy profile $\overline{\mathbf{x}}$ to be the unique solution to the following $n$ linear equations:

$$
\bar{x}_{i}=\frac{\bar{y}}{n}-\left(\gamma_{i}-\hat{\gamma}\right), \quad i \in N
$$

\footnotetext{
14 A similar statement holds with a weaker notion of payoff symmetry: if $u_{i}\left(x_{i}, y\right)=\alpha_{i}(y)+\left\langle x_{i}+\delta_{i}, \beta(y)\right\rangle$, then the sum of player payoffs can be decomposed into the sum of a function, $\Lambda(y, \bar{y})$, and a remainder, $R(\overline{\mathbf{x}})$, that is independent of $y$. Indeed, with even weaker conditions there exists a strictly monotone "welfare" function, $W: \mathbb{R}^{n} \rightarrow \mathbb{R}$, such that $W\left(u_{1}\left(y-\bar{y}+\bar{x}_{1}, y\right), \ldots, u_{n}\left(y-\bar{y}+\bar{x}_{n}, y\right)\right)$ can be written as a strictly increasing function of $\Lambda(y, \bar{y})$ that is separable from $\overline{\mathbf{x}}$.

15 There is a literature on best-response equivalences between normal-form games and identical-interest (a.k.a. best-response potential) games. Voorneveld (2000) and Morris and Ui (2004) provided several interesting results in this direction for potential games. The relationship between quasi-linear aggregate games and exact potential games is non-nested. A Cournot game with nonlinear demand but constant unit costs is a quasi-linear aggregate game, but is not an exact potential game, while a Cournot game with linear demand and nonlinear marginal cost is an exact potential game but not a quasi-linear aggregate game.

16 In terms of the complexity of solving the SGM program, note that the program in (7) can be recast as finding the minmax value of a fictitious twoperson, zero-sum game. Let player $A$ have the payoff $\Phi\left(y_{a}, y_{b}\right) \equiv \Lambda\left(y_{a}, y_{b}\right)-\Lambda\left(y_{b}, y_{b}\right)$ and player $B$ the negative of $\Phi$. By construction, it follows that

$$
\min _{y_{b} \in Y} \max _{y_{a} \in Y} \Phi\left(y_{a}, y_{b}\right) \geqslant 0 \geqslant \max _{y_{a} \in Y} \min _{y_{b} \in Y} \Phi\left(y_{a}, y_{b}\right) .
$$

The minmax solution, if one exists, corresponds to values of $\bar{y}_{a}$ and $\bar{y}_{b}$ such that $\Phi(\bar{y}, \bar{y})=0$ and $\Lambda(\bar{y}, \bar{y})=\max _{y \in Y} \Lambda(y, \bar{y})$. In this sense, when an equilibrium exists, finding the equilibrium aggregates to an $n$-player aggregate game over the full strategy space $\mathbf{X}$ is equivalent to solving a two-person zero-sum game over the smaller aggregate space, $Y$.
} 
where as before, we define $\hat{\gamma}$ as the average $\gamma_{i}$. Because $X$ is closed under addition and subtraction, we are assured that each $\bar{x}_{i}$, thus constructed, is indeed in $X$. Summing over all indices $j$ excepting $i$, we get

$$
\sum_{j \neq i} \bar{x}_{j}=\frac{n-1}{n} \bar{y}+\gamma_{i}-\hat{\gamma}, \quad i \in N .
$$

From this, we can express each player $i$ 's payoff from inducing an aggregate $y$, given the equilibrium play $\overline{\mathbf{x}}_{-i}$, as

$$
\begin{aligned}
u_{i}\left(y-\sum_{j \neq i} \bar{x}_{j}, y\right) & =u_{i}\left(y-\left(\frac{n-1}{n}\right) \bar{y}-\gamma_{i}+\hat{\gamma}, y\right) \\
& =\alpha(y)+\left\langle y-\left(\frac{n-1}{n}\right) \bar{y}-\gamma_{i}+\hat{\gamma}+\gamma_{i}, \beta(y)\right\rangle \\
& =\Lambda(y, \bar{y}) .
\end{aligned}
$$

Because $\bar{y}$ solves the SGM program in (7), it immediately follows that the constructed strategies guarantees

$$
\bar{y} \in \underset{y \in X}{\arg \max } u_{i}\left(y-\sum_{j \neq i} \bar{x}_{j}, y\right)
$$

which is the equilibrium best-response condition (4) in Lemma 1, written for the case in which $G$ is bijective. This proves that our construction of $\overline{\mathbf{x}}$ is indeed an equilibrium.

Combining our necessary and sufficient conditions above, we have our main aggregate characterization result:

Theorem 1. Suppose that $G$ is a quasi-linear aggregate game with a bijective aggregate. Then

$$
\bar{y} \in \underset{y \in X}{\arg \max } \Lambda(y, \bar{y}) \Longleftrightarrow \bar{y} \in \mathcal{E}_{Y}(G) .
$$

Condition (9) provides thus a complete characterization of the equilibrium aggregates via an SGM program. Section 3 will show the usefulness of this approach.

Invariance. Theorem 1 yields an invariance property within equivalence classes of games. Consider a family of quasi-linear, bijective aggregate games denoted by $\{G(\boldsymbol{\gamma})\}_{\boldsymbol{\gamma} \in \Gamma}$, where $\boldsymbol{\gamma}=\left(\gamma_{1}, \ldots, \gamma_{n}\right) \in \Gamma \subseteq \mathcal{V}^{n}$ parameterizes the games in terms of the players' idiosyncratic payoff components. Define an equivalence class $[\boldsymbol{\gamma}]_{\hat{\gamma}}$ of games within this family by the average $\hat{\gamma}$ :

$$
[\boldsymbol{\gamma}]_{\hat{\gamma}}=\left\{\left(\gamma_{1}, \ldots, \gamma_{n}\right) \in \mathcal{V}^{n} \mid \frac{1}{n} \sum_{i} \gamma_{i}=\hat{\gamma}\right\} .
$$

Theorem 1 states that the SGM program associated with $G(\boldsymbol{\gamma})$ provides a complete description of $\mathcal{E}_{Y}(G(\boldsymbol{\gamma}))$. We may therefore derive comparative statics on $\mathcal{E}_{Y}(G(\gamma))$ using the dual SGM-representation given by $\Lambda .{ }^{17}$ Because the SGM program associated with any game $G(\boldsymbol{\gamma})$ is invariant over the equivalence class of this game, we have an immediate neutrality result.

Proposition 1. Suppose that $\{G(\boldsymbol{\gamma})\}_{\boldsymbol{\gamma} \in \Gamma}$ is a family of bijective, quasi-linear aggregate games. Then $\mathcal{E}_{Y}(G(\boldsymbol{\gamma}))$ is invariant with respect to $[\gamma]_{\hat{\gamma}}$.

As we will see in the context of common agency games, this invariance has a surprising manifestation.

\section{Applications to intrinsic common agency games}

For the remainder of this paper, we focus our attention on characterizing the set of equilibrium aggregates for intrinsic common-agency games. Section 3.1 considers the case of adverse selection where we show that solving a simple optimization problem is enough to prove existence of non-trivial equilibria and to characterize their distortions. For the case of moral hazard, addressed in Section 3.2, we show that solving a relaxed aggregate problem again provides a proof of existence and

17 Parametric monotonicity results can be acquired in a standard manner if additional structure is placed on $\Lambda$. See, for example, Acemoglu and Jensen (2009) for a more thorough analysis of comparative statics on aggregate games with finite-dimensional strategy spaces. In the simplest and most restrictive case, let $X \subseteq \mathbb{R}$ and assume that $\Lambda(y, \bar{y}, \boldsymbol{\gamma})$ is strictly quasi-concave in $y$ and continuously differentiable in $y$ and $\boldsymbol{\gamma}$. In this case the equilibrium aggregate set, $\mathcal{E}_{Y}(G(\boldsymbol{\gamma}))$, is a singleton and, therefore, we can perform comparative statics on the object $\bar{y}(\boldsymbol{\gamma})$. Comparative statics for interior equilibria (i.e., $\bar{y} \in$ int $X$ ) may be deduced by appealing to the envelope theorem and evaluating the sign of gradient $\frac{\partial}{\partial \gamma} \Lambda_{1}(\bar{y}, \bar{y}, \boldsymbol{\gamma})$. 
a characterization of the equilibrium aggregate. These applications show the strength of the aggregate techniques to simplify the order of magnitude of the technical difficulties that one faces when looking for existence of equilibria and characterizing their properties in common agency games.

\subsection{Adverse selection}

The class of games which motivated this paper are intrinsic common agency games with public contracts where the agent has private information. ${ }^{18}$ This class is part of the broader category of distributional aggregate games as discussed above. Recall that each principal $i$ has a bounded, continuous benefit function of $v_{i}: \mathcal{Q} \rightarrow \mathbb{R}$ which gives the return associated to the agent's choice of $q \in \mathcal{Q}$ and $\mathcal{Q}$ is compact. To motivate the agent, a principal may offer a transfer schedule, $T_{i} \in \mathcal{T}$, which is an agreement to pay the agent $T_{i}(q)$ if $q \in \mathcal{Q}$ is chosen. We take the set of available contracts, $\mathcal{T}$, to be the set of bounded, upper-semicontinuous functions on $\mathcal{Q}$. Because upper-semicontinuous functions are Borel measurable, $\mathcal{T} \subseteq \mathcal{B}_{b}(\mathcal{Q})$. Principal $i$ 's payoff when the agent accepts the principal's offer and chooses $q$ is $v_{i}(q)-T_{i}(q)$. If the agent chooses not to participate, then action $q_{\emptyset}$ is implemented by default and principal $i$ earns $v_{i}\left(q_{\emptyset}\right)$, which we normalize to 0 .

We take common agency to be intrinsic in the game; i.e., the agent must either accept all contract offers (and become a common agent to all $n$ principals) or choose not to participate. In the latter case, the agent's reservation utility is normalized to $u\left(q_{\varnothing}, \theta\right)=0 .{ }^{19}$

Fixing $q(\theta, T)$, a measurable selection in the agent's best-response correspondence as in (1), we can define the distribution function ${ }^{20}$

$$
H(q \mid T) \equiv \operatorname{Prob}(\theta \in \Theta \mid q(\theta, T) \leqslant q) .
$$

Thus, taking the agent's strategy $q(\theta, T)$ as given, we have an $n$-player distributional aggregate game in which principal $i$ 's payoff is represented by

$$
u_{i}\left(T_{i}, T\right)=\int_{\mathcal{Q}}\left(v_{i}(q)-T_{i}(q)\right) d H(q \mid T) .
$$

If we characterize the set of equilibrium aggregates of this $n$-player game for every selection $q(\theta, T)$, we will have characterized the entire set of equilibrium aggregates in the original $n+1$ player game.

There are many equilibria to this game. One trivial equilibrium is for two of the $n$ principals to set transfers sufficiently negative such that every type of agent prefers to choose $q_{\emptyset}$. Such "non-participation" is an equilibrium, possibly Paretodominated by all other equilibria. We are interested in the existence of "non-trivial" equilibria in which some measure of agent types choose $q \neq q_{\emptyset}$.

Because the $n$-player game is a symmetric distributional game, we can construct an aggregate objective function following our general approach of Section 2.3.1:

$$
\Lambda(T, \bar{T})=\frac{1}{n} \int_{\mathcal{Q}}\left(\sum_{i \in N} v_{i}(q)-T(q)+(n-1)(T(q)-\bar{T}(q))\right) d H(q \mid T) .
$$

There are two cases to consider, depending upon whether or not $G$ is bijective, which in turn depends upon whether or not $\mathcal{Q}$ is finite. Consider first the case in which $\mathcal{Q}$ is a finite collection of actions, and thus a contract in $\mathcal{T}$ is a finite sequence of real numbers and the agent's best-response correspondence $Q_{0}(\theta, T)$ is non-empty. The game is bijective $T=$ $\sum_{i \in N} T_{i}$ is bijective because $\mathcal{T}$ is closed under addition and subtraction. As such, Theorem 1 applies squarely to the case of common agency games and we conclude:

Corollary 1. In the common agency game, $G$, if the action space $\mathcal{Q}$ is finite, then

$$
\mathcal{E}_{Y}(G)=\{\bar{T} \mid \bar{T} \in \underset{T \in \mathcal{T}}{\arg \max } \Lambda(T, \bar{T})\}
$$

The second case arises when $\mathcal{Q}$ is not finite. Suppose nevertheless that $\mathcal{Q}$ is a compact subset of $\mathbb{R}^{k}$, endowed with the usual topology and $\mathcal{T}$ is the set of upper-semicontinuous functions defined over $\mathcal{Q}$. Because the difference between two upper-semicontinuous functions is not necessarily upper semi-continuous, $G$ is not bijective (i.e., $\mathcal{T}$ is not closed under

\footnotetext{
18 A full treatment of the issues involved is outside the scope of this paper and is developed in Martimort and Stole (2010, 2011).

19 Note that we have not imposed any restrictions on the agent's benefit function $u$ other than continuity and boundedness; in particular, we do not impose single-crossing or monotonicity in type. Thus, the normalization of $u\left(q_{\emptyset}, \theta\right)=0$ to a type-independent reservation utility is without loss of generality as it can be embedded in the original benefit function: $\tilde{u}(q, \theta)=u(q, \theta)-u\left(q_{\emptyset}, \theta\right)$.

20 The methodological approach of using a distribution function over output which is a function of nonlinear tariffs is developed in Wilson (1993). Wilson refers to such distributions as "demand profiles".
} 
subtraction). That said, we may still apply Theorem 1 to this setting. Specializing the equilibrium construction from linear games to the present setting, we have

$$
\begin{aligned}
& \bar{T}_{i}=\frac{1}{n} \bar{T}+v_{i}-\frac{1}{n} \sum_{j \in N} v_{j}, \\
& \mathcal{T}+\left\{\bar{T}_{-i}\right\}=\mathcal{T}+\left\{\frac{n-1}{n} \bar{T}+\frac{1}{n} \sum_{j \in N} v_{j}-v_{i}\right\} .
\end{aligned}
$$

Because $\bar{T} \in \mathcal{T}$ and the principals' benefit functions are continuous, it follows that $\bar{T}_{i} \in \mathcal{T}$ and, consequently, $\bar{T} \in \mathcal{T}+\left\{\bar{T}_{-i}\right\}$. Revealed preference requires

$$
\bar{T} \in \underset{T \in \mathcal{T}}{\arg \max } \Lambda(T, \bar{T}) \Longrightarrow \bar{T} \in \underset{T \in \mathcal{T}+\left\{\bar{T}_{-i}\right\}}{\arg \max } \Lambda(T, \bar{T}) .
$$

From the sufficient conditions (8), we conclude that the constructed contracts are in fact equilibrium strategies.

Corollary 2. For the common agency game, $G$, for which $\mathcal{Q}$ is a compact subset of $\mathbb{R}^{k}$,

$$
\{\bar{T} \mid \bar{T} \in \underset{T \in \mathcal{T}}{\arg \max } \Lambda(T, \bar{T})\} \subseteq \mathcal{E}_{Y}(G)
$$

Unlike the case in which $\mathcal{Q}$ is finite, proving the reverse inclusion requires that we exploit the metric properties in the game. This proof is accomplished by showing that the difference between any pair of upper-semicontinuous functions, $T_{i}=$ $T-\bar{T}_{-i}$, can be approximated from below by another upper-semicontinuous function, $T_{i}^{\varepsilon} \nearrow T_{i}$, such that $u_{i}\left(T_{i}^{\varepsilon}, T_{i}^{\varepsilon}+\bar{T}_{-i}\right) \rightarrow$ $u_{i}\left(T_{i}, T_{i}+\bar{T}_{-i}\right)$. In words, the game is bijective on a dense subset of $\mathcal{T}$ for which payoffs are sequentially continuous. From this technical result, we have our main conclusion for intrinsic, common agency games with adverse selection.

Theorem 2. In the intrinsic common agency game, $G$, if the action space $\mathcal{Q}$ is a compact subset of $\mathbb{R}^{k}$, then

$$
\{\bar{T} \mid \bar{T} \in \underset{T \in \mathcal{T}}{\arg \max } \Lambda(T, \bar{T})\}=\mathcal{E}_{Y}(G)
$$

To give more content to this proposition, it is useful to recast the SGM program, in the standard dual action-rent formulation of the incentive literature. Let thus respectively denote by $\bar{q}(\theta) \equiv q(\theta, \bar{T}) \in \arg \max _{q \in \mathcal{Q}} u(q, \theta)+\bar{T}(q)$ and $\bar{U}(\theta) \equiv u(\bar{q}(\theta), \theta)+\bar{T}(\bar{q}(\theta))$ the equilibrium output chosen by the agent and his utility profile when the aggregate contract is $\bar{T}$.

More generally, define the set $\mathcal{I C}$ as the set of all $(q, U)$ allocations which are implementable through some aggregate transfer $T$; i.e., there exists an upper-semicontinuous $T$ such that the (measurable) output profile $q$ and the utility profile $U$ satisfy $q(\theta) \equiv q(\theta, T) \in \arg \max _{q \in \mathcal{Q}} u(q, \theta)+T(q)$ and $U(\theta) \equiv u(q(\theta), \theta)+T(q(\theta))$. Equipped with this description of implementable allocations, it is immediate to rewrite the aggregate program in Theorem 2 in a more evocative manner as:

$$
(\bar{q}, \bar{U}) \in \underset{(q, U) \in \mathcal{I C}}{\arg \max } \int_{\Theta}\left(\sum_{i \in N} v_{i}(q(\theta))-n T(q(\theta))+(n-1) \bar{T}(q(\theta))\right) d F(\theta) .
$$

Using $U(\theta)=u(q(\theta), \theta)+T(q(\theta))$, the necessary and sufficient condition (11) can be restated as:

Theorem $2^{\prime}$. The pair $(\bar{q}, \bar{U})$ comprises an equilibrium allocation if and only if

$$
\begin{aligned}
(\bar{q}, \bar{U}) \in \underset{(q, U) \in \mathcal{I} \mathcal{C}}{\arg \max } \int_{\Theta}\left(\sum_{i \in N} v_{i}(q(\theta))+u(q(\theta), \theta)-n U(\theta)\right) d F(\theta) \\
\quad+(n-1) \int_{\Theta}(u(q(\theta), \theta)+\bar{T}(q(\theta))) d F(\theta),
\end{aligned}
$$

where $\bar{T}$ implements $(\bar{q}, \bar{U})$.

The program in (12) characterizes the entire set of Nash equilibrium pairs $(\bar{q}, \bar{U})$ for intrinsic common agency games. Moreover, the characterization holds regardless of the dimension of $\Theta$ or the presence of single-crossing.

We have an immediate invariance result that is a consequence of Proposition 1. 
Corollary 3. Consider any two common agency games, $G$ and $\tilde{G}$, which differ only in the principals' payoff functions, $\left(v_{1}, \ldots, v_{n}\right)$ and $\left(\tilde{v}_{1}, \ldots, \tilde{v}_{n}\right)$, but for which the aggregate principals' payoffs are equal at each $q \in \mathcal{Q}$ :

$$
\sum_{i \in N} v_{i}(q)=\sum_{i \in N} \tilde{v}_{i}(q)
$$

Then $\mathcal{E}_{Y}(G)=\mathcal{E}_{Y}(\tilde{G})$.

Remarkably, a 2-principal game in which both principals have identical preferences and prefer higher $q$ has the same set of equilibrium aggregates as a game in which one principal prefers lower levels of $q$ and a second principal has sufficiently strong positive preference for $q$ so as to generate the same aggregate valuation.

There are many equilibria to this game. Consider first the trivial equilibria in which the agent rejects all contracts because two or more principals make undesirable offers. In the above program, this corresponds to an aggregate $\bar{T}$ that is extremely large and negative for all $q \neq q_{\emptyset}$. The solution to the self-generating maximization program therefore is to implement $q_{\emptyset}$ and the trivial outcome is an equilibrium.

Consider instead the more economically interesting equilibria in which a positive measure of agent types chooses $q \neq q_{\emptyset}$. Such equilibria will be shown to exist. Within the class of non-trivial equilibria, we focus on a natural subset in which the self-generating program collapses to a single-person maximization program. This reduction is accomplished by noting that within the allocation set $\mathcal{I C}$, we necessarily have

$$
(\bar{q}, \bar{U}) \in \underset{(q, U) \in \mathcal{I C}}{\arg \max } \int_{\Theta}(u(q(\theta), \theta)+\bar{T}(q(\theta))) d F(\theta)
$$

since by definition $\bar{q}(\theta) \in \arg \max _{q \in \mathcal{Q}} u(q, \theta)+\bar{T}(q)$.

Thus, any allocation which solves

$$
(\bar{q}, \bar{U}) \in \underset{(q, U) \in \mathcal{I C} \mathcal{C}}{\arg \max } \int_{\Theta}(W(q(\theta), \theta)-n U(\theta)) d F(\theta),
$$

where $W(q, \theta) \equiv \sum_{i \in N} v_{i}(q)+u(q, \theta)$ is the joint surplus function, will also be a solution to (12). Note that the reverse statement is decidedly untrue as the equilibrium in which two or more principals offer large negative contracts and the agent chooses not to participate does not solve (13); any solution to the program in (13) is a proper subset of the equilibrium aggregates and can be thought of as an equilibrium refinement. ${ }^{21}$ The simpler program in (13) is no longer self-generating. The absence of a fixed point allows us to conclude that non-trivial equilibria exist if an optimal solution to (13) exists, which in turn is established with minimal technical assumptions. In particular, we have the following existence result.

Theorem 3. A non-trivial equilibrium exists in the intrinsic common agency game under adverse selection if $\mathcal{Q}$ is a compact subset of $\mathbb{R}^{k}$ and either of the following conditions are satisfied:

1. $\Theta$ is finite;

2. $\Theta \subset \mathbb{R}^{k}$ is compact and convex, $u(q, \theta)=h(q)-\langle\theta, q\rangle$, and $W(\cdot, \theta)$ is concave.

Alternative assumptions which guarantee existence when $\theta$ is continuously distributed can be found in the work of Carlier (2001) and more recent generalizations to weaker assumptions by Kadan et al. (2011) which may however rely on random allocations. In the present case, however, existence is proven for deterministic equilibrium allocations; there is no role for random allocations in proving existence when $u(q, \theta)$ is bilinear in $q$.

Having established existence of solutions to (13), we can now turn to the economic properties of these simple equilibria. A cursory examination of the objective function in (13) suggests that it is as if there is a single principal maximizing collective surplus less the agent's information rent multiplied by $n$. It is tantamount to an $n$-fold marginalization of rents. A single firm collectively representing the interests of all principals would solve a similar program with $n$ replaced with 1 . Unsurprisingly, competition among the principals (weakly) reduces their payoffs relative to what they would obtain if they could maximize payoffs collectively. More surprising, however, the program implies that expected agent surplus is also lower under competition relative to the collective outcome.

Corollary 4. In every equilibrium of the intrinsic common agency game solutions to (13), the collective surplus of the principals is lower and the expected rent of the agent (across types) is weakly lower compared to the collusive outcome in which all principals collectively offer a single contract to the agent.

\footnotetext{
21 Elsewhere in Martimort and Stole (2011) we refer to this class of non-self-generating equilibria as "virtually truthful" since they are the analog to Bernheim and Whinston's (1986b) "truthful" equilibria in complete information games.
} 
We emphasize here that this conclusion is derived entirely from the character of the SGM program, without any need to calculate the solutions explicitly. This is important as the class of screening models considered here is quite large, allowing for the dimension of the parameter space to differ from that of the action space, allowing for discontinuous tariff functions, and imposing no single-crossing structure on the agent's preferences. When $\Theta$ is multi-dimensional or single-crossing fails, this approach is particularly valuable as closed-form, analytic solutions are typically elusive. ${ }^{22}$

\subsection{Moral hazard and limited liability}

As our final example, we turn to the study of intrinsic common agency games with moral hazard. This setting contrasts sharply with the previous section because it is not possible to convert the SGM program into a simpler maximization program whose solutions are also solutions to the original SGM. Thus, a direct proof of existence is elusive without more structure.

To obtain a meaningful and tractable moral-hazard agency problem, we suppose that the agent is risk neutral but is protected by limited liability. Our goal is to provide an existence theorem and a characterization of the equilibrium in an environment which thus generalizes Innes $(1990)^{23}$ but allows for competing principals. We assume that the agent chooses an effort, $e \in \mathbb{R}_{+}$, at a personal cost of $\psi(e) \geqslant 0$, with the usual technical requirements that $\psi$ is convex, $\psi(0)=\psi^{\prime}(0)=0$, and $\lim _{e \rightarrow \infty} \psi^{\prime}(e)=\infty$. Effort has the effect of stochastically improving the outcome $q$ which is distributed on the fixed support $\mathcal{Q}=\left[q_{0}, q_{1}\right]$ (with $q_{0}>0$ ). The distribution function of $q$ is denoted $F(q \mid e)$, and its associated density function is $f(q \mid e)$ which is continuous in $(q, e)$, everywhere non-negative and twice continuously differentiable in $e$. To ensure an interior solution, we shall also assume that effort has sufficiently large marginal returns at zero: i.e., $\int_{q_{0}}^{q_{1}} q f_{e}(q \mid 0) d q=$ $+\infty$. An agent who accepts an aggregate contract, $T(q)$, and chooses an effort $e$ will obtain an expected utility of

$$
\int_{\mathcal{Q}} T(q) f(q \mid e) d q-\psi(e) .
$$

To facilitate the use of the first-order approach, ${ }^{24}$ we make the simplifying assumptions of the monotone-likelihood ratio property (MLRP) and convex distribution function condition (CDFC):

$$
\frac{\partial}{\partial q}\left(\frac{f_{e}(q \mid e)}{f(q \mid e)}\right)>0 \quad \text { and } \quad F_{e e}(q \mid e) \geqslant 0 \quad \forall(q, e) \in \mathcal{Q} \times A
$$

Hence, an effort level is incentive compatible with a nondecreasing aggregate transfer $T$ if and only if

$$
\int_{q_{0}}^{q_{1}} T(q) f_{e}(q \mid e) d q-\psi^{\prime}(e)=0 .
$$

Under the stated assumptions, for any nondecreasing aggregate transfer $T$ that is not identically equal to 0 , the agent chooses a positive effort level.

We assume each of the principal's marginal value of the agent's output is constant and payoffs are linear in money, so for principal $i$ we may represent preferences over output, $q$, and transfer, $t_{i}$, as

$$
v_{i} q-t_{i}
$$

for some constant $v_{i}$. As a normalization, we assume $\sum_{i=1}^{n} v_{i}=1$ although no specific assumptions are made on the sign of each $v_{i}$. Indeed, we will see that the equilibrium aggregate is invariant to the distribution of these marginal values in accord with Proposition 1.

Limited liability imposes the following non-negativity constraint on the aggregate:

$$
T(q) \geqslant 0 .
$$

Because $\psi(0)=0,(15)$ is stronger than the ex ante participation constraint of the agent. Note, however, that this limited liability constraint only applies to the aggregate transfer and does not imply any non-negativity constraint on individual payments by the principals. Instead, each principal can undo what others are offering as long as the aggregate he wants to implement is less than the net wealth he ends up getting. ${ }^{25}$ Individually, the requirement is that the aggregate satisfies for each $i$ :

\footnotetext{
22 There are still other non-trivial equilibria that are not virtually truthful. For these equilibria, non-smooth analysis must be used to characterize the solutions to the SGM programs, which we pursue in companion papers, Martimort and Stole (2010, 2011). The approach of aggregate representation, in tandem with these tools, continues to describe the characteristics of equilibrium aggregates.

23 See also Park (1995) and Kim (1997).

24 See, for example, Rogerson (1985).

25 Any exogenous bound on a principal's payments would suffice here, so we follow the assumption in Innes (1990) for a direct comparison.
} 


$$
T(q) \leqslant v_{i} q+\bar{T}_{-i}(q) .
$$

Each principal faces the following aggregate program:

$$
\max _{T, e} \int_{\mathcal{Q}}\left(v_{i} q+\bar{T}_{-i}(q)-T(q)\right) f(q \mid e) d q
$$

subject to (14), (15) and (16).

From these individual programs, we apply our methodology and determine the equilibrium aggregate transfer $\bar{T}$ as a solution to an SGM program. We proceed as before and construct the corresponding maximand $\Lambda$ by using a change of variables and summing all individual objectives. In the same vein, summing the principals' limited liability constraints (16) implies that the aggregates $T(q)$ which are feasible deviations away from the equilibrium aggregate $\bar{T}(q)$ satisfy also the weaker condition

$$
n T(q) \leqslant q+(n-1) \bar{T}(q) .
$$

We consider a relaxed version of the SGM program in which we replace this latter constraint by an even weaker constraint which is a direct implication of (17) but where the equilibrium aggregate disappears:

$$
T(q) \leqslant q .
$$

So doing, the solutions to the SGM program are found on the larger set of possible aggregates. We will then show that the solution to this relaxed SGM program allows us to reconstruct equilibrium strategies for each principal which satisfy (16) as well.

Proceeding accordingly, we characterize the equilibrium aggregate transfer $\bar{T}$ as a solution to the following SGM program:

$$
\bar{T} \in \arg \max _{(T, e)} \Lambda(T, \bar{T}) \equiv \int_{q_{0}}^{q_{1}}\left(\frac{q}{n}+\left(1-\frac{1}{n}\right) \bar{T}(q)-T(q)\right) f(q \mid e) d q
$$

subject to (14), (15), (18).

We show in Appendix A that this problem has a solution that can be easily characterized using tools that are familiar from the monopolistic contracting literature. In particular, the equilibrium aggregate can be found in the class of contracts with a "bang-bang" nature:

$$
T(q)= \begin{cases}0 & \text { if } q \leqslant \hat{q} \\ q & \text { if } q>\hat{q}\end{cases}
$$

Let $\bar{e}(\hat{q})$ denote the agent's optimal effort when facing a contract of this form with threshold $\hat{q}$; it is implicitly defined by

$$
\int_{\hat{q}}^{q_{1}} q f_{e}(q \mid \bar{e}(\hat{q})) d q=\psi^{\prime}(\bar{e}(\hat{q}))
$$

Using the specifications in (19), we may now rewrite the non-cooperative self-generating program more simply as a program for this threshold:

$$
\bar{q} \in \arg \max _{\hat{q} \in \mathcal{Q}} \Lambda^{*}(\hat{q}, \bar{q})
$$

where

$$
\Lambda^{*}(\hat{q}, \bar{q})=\int_{q_{0}}^{\hat{q}} \frac{q}{n} f(q \mid \bar{e}(\hat{q})) d q+\left(1-\frac{1}{n}\right) \int_{\bar{q}}^{\hat{q}} q f(q \mid \bar{e}(\hat{q})) d q .
$$

Upon calculating the equilibrium threshold, $\bar{q}$, individual payments are reconstructed from the imputed $\bar{T}$, using the previous analysis and the principle of aggregate concurrence: All principals must find it optimal to implement the same effort and thus their objectives must be aligned in equilibrium. This is achieved when principal $i$ offers an equilibrium payment $\bar{T}_{i}(q)$ such that

$$
v_{i} q-\bar{T}_{i}(q)=\frac{q-\bar{T}(q)}{n} .
$$

In particular, the equilibrium so found satisfies constraint (16) for all $i$ by construction. Although our self-generating problem was based on a relaxed program, the specific nature of its solution allowed us to reconstruct an equilibrium satisfying all more stringent necessary conditions (16). 
Compare (20) with the cooperative program in which all principals combine to offer a single contract to maximize joint surplus, $W(\hat{q})$ :

$$
W(\hat{q})=\int_{q_{0}}^{\hat{q}} q f(q \mid \bar{e}(\hat{q})) d q .
$$

After scaling the objective function by $\frac{1}{n}$, it is evident that the only difference is a second term in the non-cooperative program and that the equilibrium aggregate is invariant to arbitrary redistribution of the principals' valuations for the agent's output. From this we can deduce the effect of competition on equilibrium contracts and payoffs.

The following theorem summarizes these results.

Theorem 4. An equilibrium to the intrinsic common agency game under moral hazard and limited liability exists. Any equilibrium contract threshold maximizes

$$
\bar{q} \in \arg \max _{\hat{q} \in \mathcal{Q}} \frac{W(\hat{q})}{n}+\left(1-\frac{1}{n}\right) \int_{\hat{q}}^{\bar{q}} q f(q \mid \bar{e}(\hat{q})) d q,
$$

and is invariant to the distribution of principals' preferences for output.

The agent's effort and expected payment in the non-cooperative equilibrium are less than at the cooperative solution.

Returning to the aggregate problem, direct inspection of the equilibrium conditions provides an immediate comparison between the equilibrium outcome and the cooperative outcome that would have obtained had the principals jointly designed the agent's incentive scheme. By definition of the equilibrium thresholds $\bar{q}$ and $\bar{q}^{c}$, we have:

$$
\frac{W\left(\bar{q}^{c}\right)}{n} \geqslant \frac{W(\bar{q})}{n} \geqslant \frac{W\left(\bar{q}^{c}\right)}{n}+\left(1-\frac{1}{n}\right) \int_{\bar{q}}^{\bar{q}^{c}} q f(q \mid \bar{e}(\hat{q})) d q .
$$

From which, it immediately follows that relative to the cooperative outcome $\bar{q}^{c}$, the non-cooperative equilibrium results in a higher threshold $\bar{q} .{ }^{26}$ In equilibrium, principals "on aggregate" free ride on each other and do not provide enough reward on the upper tail of the distribution. Effort and expected payments decrease.

Let us conclude by a couple of final remarks. Our "aggregate" approach may also predict the form of the equilibrium even in environments where common agency is no longer intrinsic but delegated. In a delegated common agency game, the agent chooses the subset of principals with whom to contract before uncertainty realizes. This necessarily imposes that the expected payment of each principal remains positive at the equilibrium effort; otherwise, the agent would have been better off refusing this contract. This non-negativity condition is similar to those found in Cournot games and is such that the delegated common agency games is no longer bijective.

Nevertheless, our approach can be useful for a whole ranges of cases with small asymmetries if any. Take for instance the case of symmetric principals $v_{1}=\cdots=v_{n}=\frac{1}{n}$. From (21), we have

$$
T_{i}(q)=\frac{1}{n} \bar{T}(q)
$$

and expected payments for all principals are always positive so that the equilibrium of the intrinsic game is also an equilibrium of the delegated game. ${ }^{27}$ Beyond that case, the more general analysis of delegated common agency games under moral hazard is left for further research.

\section{Appendix A}

Proof of Theorem 2. Given Corollary 2, we need only to show that

$$
\mathcal{E}_{Y}(G) \subseteq\{\bar{T} \mid \bar{T} \in \underset{T \in \mathcal{T}}{\arg \max } \Lambda(T, \bar{T})\}
$$

Suppose not. Then there is a $\bar{T} \in \mathcal{E}_{Y}(G)$ and a $\hat{T} \in \mathcal{T}$ such that

$$
\Lambda(\hat{T}, \bar{T})>\Lambda(\bar{T}, \bar{T}) .
$$

26 In fact, we prove in Appendix A that this threshold is strictly higher.

27 Martimort and Stole (2011) investigates delegated common agency games under adverse selection. 
Let $\left(\bar{T}_{1}, \ldots, \bar{T}_{n}\right)$ be the equilibrium offers which generate $\bar{T}$ and define $\hat{T}_{i} \equiv \hat{T}-\bar{T}_{-i}$. Because $\Lambda$ is the sum of the players' payoff functions, there must exist some player $i$ such that

$$
\begin{aligned}
\int_{\mathcal{Q}}\left(v_{i}(q)-\hat{T}_{i}(q)\right) d H\left(q \mid \hat{T}_{i}+\bar{T}_{-i}\right) & >\int_{\mathcal{Q}}\left(v_{i}(q)-\bar{T}_{i}(q)\right) d H\left(q \mid \bar{T}_{i}+\bar{T}_{-i}\right) \\
& =\max _{T_{i} \in \mathcal{T}} \int_{\mathcal{Q}}\left(v_{i}(q)-T_{i}(q)\right) d H\left(q \mid T_{i}+\bar{T}_{-i}\right) .
\end{aligned}
$$

The latter equality is implied by the hypothesis that $\bar{T}_{i}$ is an equilibrium strategy. Given that $\hat{T}_{i}$ yields greater payoff than $\bar{T}_{i}$, it follows that $\hat{T}_{i} \notin \mathcal{T}$.

The following lemma provides the key technical step in establishing a contradiction with the optimality of $\bar{T}_{i}$ over $\mathcal{T}$.

Lemma 3. Suppose $(\Omega, d)$ is a metric space and $\mu$ is a probability measure defined on the Borel-algebra of $\Omega$. For any bounded, lowersemicontinuous function, $f: \Omega \rightarrow \mathbb{R}$, there exists a sequence of upper-semicontinuous functions $\left(f_{m}\right)_{m \in \mathbb{N}}$ such that $f_{m}(\omega) \nearrow f(\omega)$ for each $\omega \in \Omega$, and there exists a corresponding sequence of open sets, $\left(\mathcal{O}_{m}\right)_{m \in \mathbb{N}}$, such that for each $m \in \mathbb{N}, f(\omega)=f_{m}(\omega)$ for $\omega \in \Omega \backslash \mathcal{O}_{m}$ and $\lim _{m \rightarrow \infty} \mu\left(\mathcal{O}_{m}\right)=0$.

Proof of Lemma 3. Denote the discontinuity points of $f$ by the set $\Omega_{0}$ and a typical element as $\omega_{0}$. For each such point $\omega_{0}$, we construct an $\varepsilon$-neighborhood entirely contained in $\Omega$ :

$$
B_{\varepsilon}\left(\omega_{0}\right)=\left\{\omega \in \Omega \mid d\left(\omega_{0}, \omega\right)<\varepsilon\right\},
$$

where $d$ is a metric on $\Omega$. $B_{\varepsilon}\left(\omega_{0}\right)$ is an open set in $\Omega$. Let

$$
B_{\varepsilon}=\bigcup_{\omega_{0} \in \Omega_{0}} B_{\varepsilon}\left(\omega_{0}\right)
$$

$B_{\varepsilon}$ is an open covering of $\Omega_{0}$. Remove from this set all of the discontinuity points to generate the following:

$$
\mathcal{O}_{\varepsilon}=B_{\varepsilon} \backslash \Omega_{0} .
$$

Because $\Omega_{0}$ is a closed set, $\mathcal{O}_{\varepsilon}$ is open in $\Omega$. Furthermore, $f$ is continuous on the closed complement $\Omega \backslash \mathcal{O}_{\varepsilon}$.

Construct the $\varepsilon$-approximation to $f$ as

$$
f_{\varepsilon}(\omega)= \begin{cases}f(\omega), & \omega \in \Omega \backslash \mathcal{O}_{\varepsilon} \\ \underline{f}, & \omega \in \mathcal{O}_{\varepsilon}\end{cases}
$$

where $f$ is sufficiently small so that $f<\inf _{\omega \in \mathcal{O}_{\varepsilon}} f(\omega)$. Because $f$ is bounded, such an $f$ exists. Thus, we have constructed a function that is continuous when restricted to the closed subset $\Omega \backslash \mathcal{O}_{\varepsilon}$ and also continuous when restricted to the open subset $\mathcal{O}_{\varepsilon}$. Furthermore, $f_{\varepsilon}$ is upper-semicontinuous on the boundary of these sets because the discontinuities of $f_{\varepsilon}$ are downward along any sequence $\left(\omega_{m}\right)$ traversing from $\Omega \backslash \mathcal{O}_{\varepsilon}$ to $\mathcal{O}_{\varepsilon}$. Hence, taking $\varepsilon=\frac{1}{m}$ and slightly abusing notations, the sequence $\left(f_{m}\right)$ is upper semicontinuous and converges to $f$ from below as $m \rightarrow \infty$.

By construction,

$$
\lim _{\varepsilon \rightarrow 0} \mu\left(\bigcup_{\omega \in \Omega_{0}} \mathcal{O}_{\varepsilon}(\omega)\right)=\lim _{\varepsilon \rightarrow 0} \mu\left(B_{\varepsilon}\right)-\mu\left(\Omega_{0}\right) .
$$

Also by construction, $\lim _{\varepsilon \rightarrow 0} \mu\left(B_{\varepsilon}\right)=\mu\left(\Omega_{0}\right)$ for any probability measure on the Borel-algebra of $\Omega$, so we conclude that $\lim _{\varepsilon \rightarrow 0} \mu\left(\mathcal{O}_{\varepsilon}\right)=0$. Substituting $1 / m$ for $\varepsilon$ in our construction yields $\lim _{m \rightarrow \infty} \mu\left(\mathcal{O}_{m}\right)=0$ as desired.

Returning to the main task, we wish to show that an upper-semicontinuous approximation to $\hat{T}_{i}=\hat{T}-\bar{T}_{-i}$ exists, $\hat{T}_{i}^{m}$, for which $\Lambda\left(\hat{T}_{i}^{m}+\bar{T}_{-i}, \bar{T}\right)>\Lambda\left(\bar{T}_{i}+\bar{T}_{-i}, \bar{T}\right)$. The previous lemma establishes that a sequence of approximations exists for the lower-semicontinuous function, $-\bar{T}_{-i}$, which we denote $\left(-\bar{T}_{-i}^{m}\right)_{m \in \mathbb{N}}$ (and we denote $-t=-\bar{T}_{-i}^{m}(q)$ for $q \in \mathcal{O}_{\varepsilon}$ so that is sufficiently small so that $\left.-t<\inf _{q \in \mathcal{O}_{\varepsilon}}-T_{-i}(q)\right)$. Thus, we can take $\hat{T}_{i}^{m}=\hat{T}+\left(-\bar{T}_{-i}^{m}\right)$. The resulting approximation $\hat{T}^{m}(q)=\hat{T}_{i}^{m}(q)+\bar{T}_{-i}(q)$ is equal to $\hat{T}(q)$ for all $q \in \mathcal{Q}$ excepting the open set $\mathcal{O}_{m}$, which can be made arbitrarily small. Because $\hat{T}^{m} \leqslant \hat{T}$ with equality on $q \notin \mathcal{O}_{m}$, revealed preference implies

$$
\bar{q}_{0}(\theta, \hat{T}) \notin \mathcal{O}_{m} \quad \Rightarrow \quad \bar{q}_{0}\left(\theta, \hat{T}^{m}\right)=\bar{q}_{0}(\theta, \hat{T}) .
$$

Because $\mu\left(\mathcal{O}_{m}\right) \rightarrow 0$, we have pointwise convergence $\bar{q}_{0}\left(\theta, \hat{T}^{m}(q)\right) \rightarrow \bar{q}_{0}(\theta, \hat{T}(q))$, 
$\operatorname{Prob}\left(\theta \in \Theta \mid \bar{q}_{0}(\theta, \hat{T}) \in \mathcal{O}_{m}\right) \rightarrow 0$,

and $H\left(\cdot \mid \hat{T}^{m}\right)$ converges in distribution to $H(\cdot \mid \hat{T})$.

Consider the limiting payoff to player $i$ from playing $\hat{T}_{i}^{m} \in \mathcal{T}$ :

$$
\liminf _{m \rightarrow \infty} \int_{\mathcal{Q}}\left(v_{i}-\hat{T}_{i}^{m}\right) d H\left(q \mid \hat{T}_{i}^{m}+\bar{T}_{-i}\right) .
$$

The first component is immediate from the convergence of distributions.

$$
\int_{\mathcal{Q}} v_{i}(q) d H\left(q, \hat{T}^{m}\right) \rightarrow \int_{\mathcal{Q}} v_{i}(q) d H(q, \hat{T}) .
$$

Now consider the second term,

$$
\int_{\mathcal{Q}}-\hat{T}_{i}^{m}(q) d H\left(q \mid \hat{T}^{m}\right)=t \int_{\mathcal{O}_{m}} d H\left(q \mid \hat{T}^{m}\right)+\int_{\mathcal{Q} \backslash \mathcal{O}_{m}}-\hat{T}_{i}(q) d H\left(q \mid \hat{T}^{m}\right) .
$$

Convergence in distributions implies that

$$
\lim _{m \rightarrow \infty} \int_{\mathcal{O}_{m}} d H\left(q \mid \hat{T}^{m}\right)=0
$$

Because $-\hat{T}_{i}$ is lower-semicontinuous on $\mathcal{Q} \backslash \mathcal{O}_{m}$, the Portmanteau theorem provides that

$$
\liminf \int_{\mathcal{Q} \backslash \mathcal{O}_{m}} \hat{T}_{i}(q) d H\left(q \mid \hat{T}^{m}\right) \geqslant \int_{\mathcal{Q} \backslash \mathcal{O}_{m}} \hat{T}_{i}(q) d H(q \mid \hat{T}) .
$$

Thus, we have established there exists a sequence $\hat{T}_{i}^{m} \in \mathcal{T}$ such that

$$
\begin{aligned}
\liminf \int_{\mathcal{Q}}\left(v_{i}(q)-\hat{T}_{i}^{m}(q)\right) d H\left(q \mid \hat{T}_{i}^{m}+\bar{T}_{-i}\right) & \geqslant \int_{\mathcal{Q}}\left(v_{i}(q)-\hat{T}_{i}(q)\right) d H\left(q \mid \hat{T}_{i}+\bar{T}_{-i}\right) \\
& >\int_{\mathcal{Q}}\left(v_{i}(q)-\bar{T}_{i}(q)\right) d H\left(q \mid \bar{T}_{i}+\bar{T}_{-i}\right),
\end{aligned}
$$

which contradicts the hypothesis that $\bar{T}_{i}$ is a best response to $\bar{T}_{-i}$ over the set of bounded, upper-semicontinuous functions, $\mathcal{T}$.

Proof of Theorem 3. Define the upper bound on total surplus relative to no contract,

$$
\bar{W} \equiv \max _{q \in \mathcal{Q}, \theta \in \Theta} W(q, \theta)-W\left(q_{\emptyset}, \theta\right)
$$

Consider the program in (13) with the additional constraint that the agent's rent is bounded by $\bar{W}$. Note that giving the agent all of the surplus is feasible, but dominated by the null contract. Hence, if there is a solution to (13), then the agent's rent term must lie below this upper bound. It therefore suffices to prove a solution exists to the constrained program.

Because $\mathcal{Q}$ is a compact subset of $\mathbb{R}^{k}$ and $[0, \bar{W}]$ is compact in $\mathbb{R}$, if $\Theta$ is finite then the program in (13) is over a finite-dimension, compact set. Because the payoff functions are continuous, a solution exists from the Weirstrass Theorem.

Suppose instead that $\theta$ is distributed on a bounded, connected set $\Theta \subset \mathbb{R}^{k}$, but that $u(q, \theta)$ is bilinear in $q$ and $\theta$. Such linearity implies that the set of feasible allocations, $I C$, can be identified with the space of convex indirect utility functions given by $\mathcal{U}$. In particular, we can take $\mathcal{U}$ to be the set of convex functions mapping from $\Theta$ to $[0, \bar{W}]$ for which $U$ and $\nabla U$ are square integrable, i.e., $\mathcal{U} \subset \mathcal{H}^{1}(\Theta){ }^{28}$ Define the objective in (13) as a function of $U$ as

$$
\Pi(U) \equiv \int_{\Theta}(W(\nabla U(\theta), \theta)-n U(\theta)) d F(\theta) .
$$

\footnotetext{
$28 \mathcal{H}^{1}$ is a Hilbert space with norm $\|U\|_{\mathcal{H}^{1}}=\int_{\Theta}\left(U^{2}(\theta)+\|\nabla U(\theta)\|^{2}\right) d \theta$. In some functional analysis textbooks, $\mathcal{H}^{1}$ is denoted $\mathcal{H}^{1,2}$ or, using the notation of Sobolev spaces, $W^{1,2}$. See Jost (2005), Corollary 20.10, for a formal proof of the equivalence of $\mathcal{H}^{1}$ and the Sobolev space $W^{1,2}$.
} 
Because convex functions are almost everywhere differentiable, $\Pi(U)$ is well defined on $\mathcal{U}$. Existence is proved by demonstrating that the constrained program consists of maximizing a weakly upper-semicontinuous function over a weakly compact set. To establish weak upper-semicontinuity, note that $\Pi$ is concave in $U$. This follows immediately from the assumption that $W$ is concave in $\nabla U$ and that $\mathcal{U}$ is a convex space of functions. $\Pi$ contains two components. Using Theorem 22.4 from Jost (2005), the first component (the integral of $W$ ) is (strongly) upper semicontinuous. The second component (the integral of $n U$ ) is a bounded linear functional and hence strongly continuous. We conclude that $\Pi$ is both concave and strongly upper-semicontinuous in $U$. Using Lemma 22.6 from Jost (2005), concavity and strong u.s.c. implies that $\Pi$ is also weakly upper-semicontinuous. The second step is to note that $\mathcal{U}$ is bounded, convex and closed. Boundedness and convexity are immediate by construction. Closedness follows from a result found in Rochet and Chone (1998, p. 819) that the limit of a sequence of convex functions is convex using the $\mathcal{H}^{1}$ norm. We can therefore apply a version of the James' Theorem to conclude that $\mathcal{U}$ is weakly sequentially compact. (See, for example, Jahn (2007), Appendix B.) We may now invoke the weak-topology variation of the Weirstrass Theorem (see, for example, Jahn (2007), Theorem 2.12), and conclude a solution to the program exists.

Proof of Theorem 4. First, we prove a useful lemma, that is useful both in the cooperative and the non-cooperative outcomes. Consider the following program:

$$
\left(\mathcal{P}_{n}\right): \max _{T, e} \int_{q_{0}}^{q_{1}}\left(\frac{q}{n}+\left(1-\frac{1}{n}\right) \hat{T}(q)-T(q)\right) f(q \mid e) d q
$$

subject to (14), (15), (18).

Let $(\bar{T}, \bar{e})$ be the solution to this problem. Importantly, $\left(\mathcal{P}_{n}\right)$ covers both the case of an equilibrium with $n>1$ agents by setting $\bar{T}=\hat{T}$ and the cooperative solution in which $n=1$.

Lemma 4. $\left(\mathcal{P}_{n}\right)$ admits a "bang-bang" solution of the form given by (19).

Proof. Let us form the Lagrangean for this program (omitting constraints (15) and (18) for simplicity):

$$
\mathcal{L}_{n}(T, e)=\int_{q_{0}}^{q_{1}}\left(\frac{q}{n}+\left(1-\frac{1}{n}\right) \hat{T}(q)-T(q)\right) f(q \mid e) d q+\lambda\left(\int_{q_{0}}^{q_{1}} T(q) f_{e}(q \mid e) d q-\psi^{\prime}(e)\right)
$$

where $\lambda$ is the multiplier of the incentive constraint (14).

Because the objective is linear in $T(q), T(q)$ takes on only one of two values on the extremes of the constrained set: $T(q)=0$ or $T(q)=q$. In particular, the coefficient of $T(q)$ in the Lagrangean is

$$
-f(q \mid e)+\lambda f_{e}(q \mid e) .
$$

Define $\hat{q}$ as $\lambda f_{e}(\hat{q} \mid e)=f(\hat{q} \mid e)$. From MLRP, the above coefficient is positive if $q \geqslant \hat{q}$ (respectively, $\left.q<\hat{q}\right)$ if $\lambda \geqslant 0$. This implies that $T(q)=q$ (respectively, $T(q)=0$ ) for $q \geqslant \hat{q}$ (respectively, $q<\hat{q}$ ) and the optimal payment schedule is as in (19).

In fact, we have $\lambda \geqslant 0$ when $\bar{e}>0$. The optimality of the Lagrangean and the fact that payments are non-negative altogether imply $\lambda \bar{T}(q) f_{e}(q \mid \bar{e})-\bar{T}(q) f(q \mid \bar{e}) \geqslant 0$ were $\bar{T}$ is the solution to the optimization program and $\bar{e}$ the corresponding effort. Integrating and taking into account (14) yields:

$$
\lambda \psi^{\prime}(\bar{e}) \geqslant \int_{q_{0}}^{q_{1}} \bar{T}(q) f(q \mid \bar{e}) d q \geqslant 0
$$

where last inequality follows from the non-negativity of payments. If $\bar{e}>0$, we immediately get $\lambda \geqslant 0$. Hence, the solution to $\left(\mathcal{P}_{n}\right)$ is "bang-bang" if it implements a positive effort.

Suppose on the other hand that $\bar{e}=0$, then a zero payment which belongs to the class defined in (19) also implements that effort which ends the proof.

Simplified incentive constraint. Being given that we can look for the optimal contract in the class given by (19), the incentive constraint can be written as

$$
\mathcal{G}(e, \hat{q})=\int_{\hat{q}}^{q_{1}} q f_{e}(q \mid e) d q-\psi^{\prime}(e)=0
$$


By quasi-concavity of the agent's problem, Eq. (22) defines a unique solution $\bar{e}(\hat{q})$. We have $\frac{\partial \mathcal{G}}{\partial e}(e, \hat{q})=\int_{\hat{q}}^{q_{1}} q f_{e e}(q \mid e) d q-$ $\psi^{\prime \prime}(e)<0$ from CDFC and strict convexity of $\psi(\cdot)$. Moreover, we also have $\frac{\partial \mathcal{G}}{\partial \hat{q}}(e, \hat{q})=-\hat{q} f_{e}(\hat{q} \mid e)$. From the Implicit Function Theorem, $\bar{e}(\hat{q})$ is differentiable in $\hat{q}$ and

$$
\dot{\bar{e}}(\hat{q})=-\frac{\frac{\partial \mathcal{G}}{\partial \hat{q}}(\bar{e}(\hat{q}), \hat{q})}{\frac{\partial \mathcal{G}}{\partial e}(\bar{e}(\hat{q}), \hat{q})}=\frac{\hat{q} f_{e}(\hat{q} \mid \bar{e}(\hat{q}))}{\int_{\hat{q}}^{q_{1}} q f_{e e}(q \mid \bar{e}(\hat{q})) d q-\psi^{\prime \prime}(\bar{e}(\hat{q}))} .
$$

Observe that $\bar{e}\left(q_{0}\right)$ is the first-best level of effort and $\frac{f\left(q_{0} \mid \bar{e}\left(q_{0}\right)\right)}{f_{e}\left(q_{0} \mid \bar{e}\left(q_{0}\right)\right)}<0$ from MLRP, so that $\dot{\bar{e}}\left(q_{0}\right)>0$. Similarly, we have $\bar{e}\left(q_{1}\right)=0, \frac{f\left(q_{1} \mid 0\right)}{f_{e}\left(q_{1} \mid 0\right)}>0$ from MLRP, $\frac{\partial \mathcal{G}}{\partial e}\left(0, q_{1}\right)=-\psi^{\prime \prime}(0)$ so that $\dot{\bar{e}}\left(q_{1}\right)<0$.

Cooperative setting. We now develop as a useful benchmark the solution to the cooperative program had principals cooperated in dealing with the agent. This will provide us a condition under which the equilibrium effort is positive. Then, we turn to the non-cooperative setting.

The optimal contract $\bar{T}^{c}$ jointly offered by principals solves $\left(\mathcal{P}_{1}\right)$ and thus is implementable through a threshold $\bar{q}^{c}$. With contracts in the class (19), the principals' expected payoff becomes

$$
W(\hat{q})=\int_{q_{0}}^{\hat{q}} q f(q \mid \bar{e}(\hat{q})) d q .
$$

We immediately obtain

$$
\dot{W}(\hat{q})=\hat{q} f(\hat{q} \mid \bar{e}(\hat{q}))+\left(\int_{q_{0}}^{\hat{q}} q f_{e}(q \mid \bar{e}(\hat{q})) d q\right) \dot{\bar{e}}(\hat{q}) .
$$

It follows that

$$
\dot{W}\left(q_{0}\right)=q_{0} f\left(q_{0} \mid \bar{e}\left(q_{0}\right)\right)>0,
$$

and

$$
\dot{W}\left(q_{1}\right)=\frac{q_{1} f_{e}\left(q_{1} \mid 0\right)}{\psi^{\prime \prime}(0)}\left(\frac{f\left(q_{1} \mid 0\right)}{f_{e}\left(q_{1} \mid 0\right)} \psi^{\prime \prime}(0)-\int_{q_{0}}^{q_{1}} q f_{e}(q \mid 0) d q\right)<0
$$

when $\int_{q_{0}}^{q_{1}} q f_{e}(q \mid 0) d q=+\infty$ as assumed.

We conclude that there exists an interior maximum of $W(\hat{q})$ at $\bar{q}^{c} \in\left(q_{0}, q_{1}\right)$ such that:

$$
\dot{W}\left(q^{c}\right)=\bar{q}^{c} f\left(\bar{q}^{c} \mid \bar{e}\left(\bar{q}^{c}\right)\right)+\left(\int_{q_{0}}^{\bar{q}^{c}} q f_{e}\left(q \mid \bar{e}\left(\bar{q}^{c}\right)\right) d q\right) \dot{\bar{e}}\left(\bar{q}^{c}\right)=0 .
$$

The corresponding effort $\bar{e}\left(\bar{q}^{c}\right)$ is thus positive.

Non-cooperative setting. We prove existence by showing that a solution to the relaxed SGM program exists, and that such a solution is indeed an equilibrium.

The solution $\bar{T}$ to the self-generating program solves $\left(\mathcal{P}_{n}\right)$ with $\bar{T}=\hat{T}$. It is thus is implementable through a threshold $\bar{q}$. With contracts in the class (19), the SGM program can be rewritten as (20).

Observe now that $\Lambda^{*}(\hat{q}, \bar{q})$ is differentiable in $\hat{q}$ with a derivative

$$
\frac{\partial \Lambda^{*}}{\partial \hat{q}}(\hat{q}, \bar{q})=\hat{q} f(\hat{q} \mid \bar{e}(\hat{q}))+\left(\frac{1}{n} \int_{q_{0}}^{\hat{q}} q f_{e}(q \mid \bar{e}(\hat{q})) d q+\left(1-\frac{1}{n}\right)\left(\int_{\bar{q}}^{\hat{q}} q f_{e}(q \mid \bar{e}(\hat{q})) d q\right)\right) \dot{\bar{e}}(\hat{q}) .
$$

First, notice that

$$
\frac{\partial \Lambda^{*}}{\partial \hat{q}}\left(q_{0}, q_{0}\right)=q_{0} f_{e}\left(q_{0} \mid \bar{e}(0)\right)>0 .
$$

Increasing $\hat{q}$ above $q_{0}$ increases $\Lambda^{*}\left(\hat{q}, q_{0}\right)$ so that $q_{0}$ is not a possible equilibrium aggregate. Also, the assumption $\int_{q_{0}}^{q_{1}} q f_{e}(q \mid 0) d q=+\infty$ implies: 


$$
\frac{\partial \Lambda^{*}}{\partial \hat{q}}\left(q_{1}, q_{1}\right)=\frac{q_{1} f_{e}\left(q_{1} \mid 0\right)}{\psi^{\prime \prime}(0)}\left(\frac{f\left(q_{1} \mid 0\right)}{f_{e}\left(q_{1} \mid 0\right)} \psi^{\prime \prime}(0)-\frac{1}{n} \int_{q_{0}}^{q_{1}} q f_{e}(q \mid 0) d q\right)<0 .
$$

Decreasing $\hat{q}$ below $q_{1}$ thus increases $\Lambda\left(\hat{q}, q_{1}\right)$ and $q_{1}$ is not an equilibrium aggregate either.

Any interior solution $\bar{q}$ to the SGM program must thus satisfy

$$
\frac{\partial \Lambda^{*}}{\partial \hat{q}}(\bar{q}, \bar{q})=0=\bar{q} f(\bar{q} \mid \bar{e}(\bar{q}))+\left(\frac{1}{n} \int_{q_{0}}^{\bar{q}} q f_{e}(q \mid \bar{e}(\bar{q})) d q\right) \dot{\bar{e}}(\bar{q})=0 .
$$

From the Theorem of Intermediary Values, such a solution exists since $\frac{\partial \Lambda}{\partial \hat{q}}(\bar{q}, \bar{q})$ is continuous in $\bar{q}$, positive at $q_{0}$ and negative at $q_{1}$.

Inserting (24) into (25) yields

$$
\frac{\partial \Lambda^{*}}{\partial \hat{q}}\left(\bar{q}^{c}, \bar{q}^{c}\right)=\left(1-\frac{1}{n}\right) \bar{q}^{c} f\left(\bar{q}^{c} \mid \bar{e}\left(\bar{q}^{c}\right)\right)>0 .
$$

This proves that $\bar{q} \neq \bar{q}^{c}$. From a remark in the text, it immediately follows that in fact any equilibrium $\bar{q}$ satisfies $\bar{q}>\bar{q}^{c}$.

Equilibrium payments. Having established that $\bar{T}$ satisfies the relaxed program, we construct individual transfers as in the text:

$$
\bar{T}_{i}(q)=v_{i} q+\frac{\bar{T}(q)-q}{n}
$$

Hence,

$$
v_{i} q+\bar{T}_{-i}(q)=\frac{q}{n}+\left(\frac{n-1}{n}\right) \bar{T}(q)
$$

and each principal's program is equivalent to the objective in the aggregate program. Because these transfers also satisfy the individual principal constraints in (16), the aggregate $\bar{T}$ also is a solution to the full SGM program.

Comparative statics. We first prove the following lemmas.

Lemma 5. $\bar{e}(\hat{q})$ is quasi-concave in $\hat{q}$ with an interior maximum at $q^{*}$.

Proof. We already noticed that $\dot{\bar{e}}\left(q_{0}\right)>0>\dot{\bar{e}}\left(q_{1}\right)$. Let now $q^{*}$ be any interior solution to $\dot{\bar{e}}\left(q^{*}\right)=0$. It satisfies $f_{e}\left(q^{*} \mid \bar{e}\left(q^{*}\right)\right)=$ 0 . From MLRP, we have $f_{e}\left(q \mid \bar{e}\left(q^{*}\right)\right)>f_{e}\left(q^{*} \mid \bar{e}\left(q^{*}\right)\right)=0$ for $q>q^{*}$. This implies that, for $\hat{q}>q^{*}$,

$$
\int_{\hat{q}}^{q_{1}} q f_{e}\left(q \mid \bar{e}\left(q^{*}\right)\right) d q<\int_{q^{*}}^{q_{1}} q f_{e}\left(q \mid \bar{e}\left(q^{*}\right)\right) d q=\psi^{\prime}\left(\bar{e}\left(q^{*}\right)\right)
$$

where the last equality follows from of definition of $\bar{e}\left(q^{*}\right)$. Because, the agent's problem is quasi-concave in $e$ when offered $\hat{q}$, we finally have $\bar{e}\left(q^{*}\right)>\bar{e}(\hat{q})$ which shows that $\bar{e}$ is decreasing on the right of $q^{*}$. A symmetric argument shows that $\bar{e}$ is increasing on the left of $q^{*}$ which ends the proof.

Lemma 6. $\dot{\bar{e}}\left(\bar{q}^{c}\right) \leqslant 0$.

Proof. Inserting the value of $\dot{\bar{e}}\left(\bar{q}^{c}\right)$ obtained from (23) into (24) gives following equality:

$$
f\left(\bar{q}^{c} \mid \bar{e}\left(\bar{q}^{c}\right)\right)=\frac{f_{e}\left(\bar{q}^{c} \mid \bar{e}\left(\bar{q}^{c}\right)\right) \int_{q_{0}}^{\bar{q}^{c}} q f_{e}\left(q \mid \bar{e}\left(\bar{q}^{c}\right)\right) d q}{\int_{\bar{q}^{c}}^{q_{1}} q f_{e e}\left(q \mid \bar{e}\left(\bar{q}^{c}\right)\right) d q-\psi^{\prime \prime}\left(\bar{e}\left(\bar{q}^{c}\right)\right)} .
$$

Suppose that $\bar{q}^{c} \leqslant q^{*}$, or from the quasi-concavity of $\bar{e}$ (Lemma 5), $\dot{\bar{e}}\left(\bar{q}^{c}\right) \geqslant 0$ which amounts to $f_{e}\left(\bar{q}^{c} \mid \bar{e}\left(\bar{q}^{c}\right)\right) \leqslant 0$. From MLRP, we get then $f_{e}\left(q \mid \bar{e}\left(\bar{q}^{c}\right)\right)<0$ for all $q \leqslant \bar{q}^{c}$ and thus $\int_{q_{0}}^{\bar{q}^{c}} q f_{e}\left(q \mid \bar{e}\left(\bar{q}^{c}\right)\right) d q<0$. But this implies that the right-hand side of (27) is non-positive; a contradiction with the positive sign of the left-hand side.

Using Lemmas 5 and 6, it follows that $\bar{e}(\bar{q})<\bar{e}\left(\bar{q}^{c}\right)$. Finally, the agent's expected payments is lower under the noncooperative solution. 


\section{References}

Acemoglu, D., Jensen, M., 2009. Aggregate comparative statics. Working paper.

Aliprantis, C., Border, K., 2006. Infinite Dimensional Analysis: A Hitchhiker’s Guide, 3rd edition. Springer, Berlin.

Bergstrom, T., Blume, L., Varian, H., 1986. On the private provision of public goods. J. Publ. Econ. 29, 25-49.

Bernheim, D., Whinston, M., 1986a. Common agency. Econometrica 54, 923-942.

Bernheim, D., Whinston, M., 1986b. Menu auctions, resource allocations and economic influence. Quart. J. Econ. 101, 1-31.

Carlier, G., 2001. A general existence result for the principal-agent problem with adverse selection. J. Math. Econ. 35, 129-150.

Carmona, G., Fajardo, J., 2009. Existence of equilibrium in common agency games with adverse selection. Games Econ. Behav. 66, 749-760.

Corchon, L., 1994. Comparative statics for aggregative games: The strong concavity case. Math. Soc. Sci. 28, 151-165.

Cornes, R., Hartley, R., 2000. Joint production games and share functions. Working paper.

Cornes, R., Hartley, R., 2005. Asymmetric contests with general technologies. Econ. Theory 26, 923-936.

Dixit, A., 1996. The Making of Economic Policy. MIT Press, Cambridge.

Dubey, P., Haimanko, O., Zapehelnyuk, A., 2006. Strategic complements and substitutes, and potential games. Games Econ. Behav. 54, 77-94.

Fraysse, J., 1993. Common agency: Existence of an equilibrium in the case of two outcomes. Econometrica 61, 1225-1229.

Gentzkow, M., Kamenica, E., 2011. Persuasion games. Working paper, University of Chicago Booth School of Business, March 2011.

Holmström, B., Milgrom, P., 1988. Common agency and exclusive dealing. Unpublished manuscript, Northwestern University.

Innes, R., 1990. Limited liability and incentive contracting with ex-ante action choices. J. Econ. Theory 52, 47-65.

Jahn, J., 2007. Introduction to the Theory of Nonlinear Optimization, 3rd edition. Springer-Verlag, Berlin.

Jensen, M., 2010. Aggregative games and best-reply potentials. Econ. Theory 43, 45-66.

Jost, J., 2005. Postmodern Analysis, 3rd edition. Springer-Verlag, Berlin.

Kadan, O., Reny, P., Swinkels, J., 2011. Existence of optimal mechanisms in principal-agent problems. Working paper.

Kim, S.K., 1997. Limited liability and bonus contracts. J. Econ. Manag. Strategy 6, 899-913.

Klemperer, P., Meyer, M., 1989. Supply function equilibria in oligopoly under uncertainty. Econometrica 57, 1243-1277.

Kukushkin, N., 1994. A fixed-point theorem for decreasing mappings. Econ. Letters 46, 23-26.

Kukushkin, N., 2004. Best response dynamics in finite games with additive aggregation. Games Econ. Behav. 48, 94-110.

Martimort, D., 1992. Multi-principaux avec anti-selection. Ann. Econ. Statist. 28, 1-38.

Martimort, D., Stole, L., 2002. The revelation and delegation principles in common agency games. Econometrica 70, $1659-1674$.

Martimort, D., Stole, L., 2010. Public contracting in intrinsic common-agency games. Revision of "Common agency games with common screening devices", 2005. Working paper.

Martimort, D., Stole, L., 2011. Public contracting in delegated agency games. Working paper.

Monteiro, P., Page, F., 2005. Existence of Nash equilibrium in competitive nonlinear pricing games with adverse selection. Mimeo, Fundacao Getulio Vargas and University of Alabama.

Morris, S., Ui, T., 2004. Best response equivalence. Games Econ. Behav. 49, 260-287.

Novshek, W., 1984. Finding all $n$-firm Cournot equilibria. Int. Econ. Rev. 25, 61-70.

Novshek, W., 1985. On the existence of Cournot equilibrium. Rev. Econ. Stud. 52, 85-98.

Okuguchi, K., 1993. Unified approach to Cournot models: oligopoly, taxation and aggregate provision of a pure public good. Europ. J. Polit. Economy 9, 233-245.

Page, F., 2008. Catalog competition and stable nonlinear prices. J. Math. Econ. 44, 822-835.

Page, F., Monteiro, P., 2003. Three principles of competitive nonlinear pricing. J. Math. Econ. 39, 63-109.

Park, E.-S., 1995. Incentive contracting under limited liability. J. Econ. Manag. Strategy 4, 477-490.

Peters, M., 2001. Common agency and the revelation principle. Econometrica 69, 1349-1372.

Rochet, J.-C., Chone, P., 1998. Ironing, sweeping and multidimensional screening. Econometrica 66, 783-826.

Rogerson, W., 1985. The first-order approach to principal-agent problems. Econometrica 53, 1357-1367.

Selten, R., 1970. Preispolitik der Mehrproduktenunternehmung in der statischen Theorie. Springer-Verlag, Berlin.

Simon, L., Zame, W., 1990. Discontinuous games and endogenous sharing rules. Econometrica 58, 861-872.

Stole, L., 1991. Mechanism design under common agency. Unpublished manuscript, University of Chicago.

Szidarovszky, F., Yakowitz, S., 1977. A new proof of the existences and uniqueness of the Cournot equilibrium. Int. Econ. Rev. 18, 787-789.

Voorneveld, M., 2000. Best-response potential games. Econ. Letters 66, 289-295.

Wilson, R., 1993. Nonlinear Pricing. Oxford University Press. 